ARTICLE

\title{
VEGFR2 signaling drives meningeal vascular regeneration upon head injury
}

Bong Ihn Koh (1) 1, Hyuek Jong Lee ${ }^{2}$, Pil Ae Kwak ${ }^{2}$, Myung Jin Yang ${ }^{2,3}$, Ju-Hee Kim³ ${ }^{3}$ Hyung-Seok Kim ${ }^{4}$ Gou Young Koh (1) ${ }^{1,2,3,5 凶}$ \& Injune Kim (1) ${ }^{1,3,5 凶}$

Upon severe head injury $(\mathrm{HI})$, blood vessels of the meninges and brain parenchyma are inevitably damaged. While limited vascular regeneration of the injured brain has been studied extensively, our understanding of meningeal vascular regeneration following head injury is quite limited. Here, we identify key pathways governing meningeal vascular regeneration following $\mathrm{HI}$. Rapid and complete vascular regeneration in the meninges is predominantly driven by VEGFR2 signaling. Substantial increase of VEGFR2 is observed in both human patients and mouse models of $\mathrm{HI}$, and endothelial cell-specific deletion of Vegfr 2 in the latter inhibits meningeal vascular regeneration. We further identify the facilitating, stabilizing and arresting roles of Tie2, PDGFR $\beta$ and DII4 signaling, respectively, in meningeal vascular regeneration. Prolonged inhibition of this angiogenic process following $\mathrm{HI}$ compromises immunological and stromal integrity of the injured meninges. These findings establish a molecular framework for meningeal vascular regeneration after $\mathrm{HI}$, and may guide development of wound healing therapeutics.

\footnotetext{
${ }^{1}$ KI for Bio-century, Korea Advanced Institute of Science and Technology (KAIST), Daejeon 34141, Republic of Korea. ${ }^{2}$ Center for Vascular Research, Institute for Basic Science (IBS), Daejeon 34141, Republic of Korea. ${ }^{3}$ Graduate School of Medical Science and Engineering, Korea Advanced Institute of Science and Technology, Daejeon 34141, Republic of Korea. ${ }^{4}$ Department of Forensic Medicine, Chonnam National University Medical School, Gwangju 61463, Republic of Korea. ${ }^{5}$ These authors jointly supervised this work: Gou Young Koh, Injune Kim. ${ }^{凶}$ email: gykoh@kaist.ac.kr; injunek@kaist.ac.kr
} 
$\mathrm{T}$ raumatic brain injuries (TBIs) arising from various accidents, ranging from vehicle-related collisions to sports injuries, are the most frequent form of brain injury in humans ${ }^{1,2}$. TBI may cause a wide range of health issues from behavioral changes to pronounced cognitive dysfunction ${ }^{3-5}$. As TBI causes injury to both the brain parenchyma (hereafter, referred to as "brain") and neighboring meningeal blood vessels (BVs), such injuries are better considered as head injuries (HIs) with respect to all the cranial tissues affected by the physical impact.

The meninges are composed of the dura mater, arachnoid membrane, subarachnoid space, and pia mater, all of which protect the brain ${ }^{6,7}$. The dura mater is highly vascularized with precapillary arterioles, capillary beds, and postcapillary venules ${ }^{8,9}$, of which the latter two vessel types support immune cell trafficking and surveillance for the protection of the central nervous system ${ }^{10}$.

Although a plethora of studies has investigated the molecular mechanisms underlying vascular responses in the brain following $\mathrm{TBI}^{11-13}$, responses to such inevitable injuries in the neighboring meninges remain largely unexplored. A recent report indicates that, upon compression injury to the skull, injured meningeal BVs undergo rapid regeneration with the aid of different macrophage subsets $^{14}$. Although this previous study demonstrates the myeloid cells' ability to break down physical obstructions in the angiogenic path, it does not identify that fundamental angiogenic pathways contribute to the rapid regeneration of injured meningeal BVs.

In this study, we investigate which underlying molecular and cellular mechanisms drive vascular regeneration in the dura mater. We provide a comprehensive molecular characterization of dura mater BVs both in homeostasis and following vascular injury, highlighting fundamental differences between dura mater and brain endothelial cells (ECs) and thereby suggesting differential organotypic treatment for HI care.

\section{Results}

Dynamic vascular regeneration occurs in dura mater after PTI. We employed photothrombotic injury (PTI) ${ }^{15}$ as an $\mathrm{HI}$ model to inflict vascular damage simultaneously onto the dura mater, pia mater, and brain of mice and compared the vascular changes in these compartments (Fig. 1a-c). Vascular responses to PTI were different for each compartment (Fig. 1d-g). In the dura mater, BVs re-vascularized avascular areas swiftly and almost completely regenerated to its original state at 7 days after PTI (D7) (Fig. 1d, e). Given that EdU-incorporated proliferative ECs formed typical sprouts at the vascular front of the recovering dura mater at D3 (Fig. $1 \mathrm{~h}, \mathrm{i}$ ), vascular regeneration seemed to be mediated mainly by active sprouting angiogenesis. In the pia mater, the injured BVs undertook extensive vascular remodeling, including active sprouting angiogenesis highlighted by numerous vascular sprouts and proliferative ECs at D3 (Fig. 1i), which led to excessive vascular content with abnormally dilated BVs at D7 (Fig. 1d, f). In contrast, the brain vessels exhibited the poorest rate of regeneration, predominantly showing vascular collapse, substantial vascular dilation, and large avascular areas at D7 (Fig. 1d, g). Vascular sprouts and EdU-incorporated proliferative ECs were rarely found in the brain at D3 (Fig. 1i). Lectin perfusion analysis revealed that the recovered BVs exhibited functional blood flow by $80-90 \%$ and $92-98 \%$ at D7 and D14 in the dura mater (Supplementary Fig. 1a, b). In contrast, regeneration of blood perfusion was rarely seen in the brain (Supplementary Fig. 1c), highlighting the superior functional regeneration of dura mater BVs following PTI.

PTI causes minor and transient vascular leakage in dura mater. Edema caused by vascular leakage and tissue death can cause severe complications in patients with $\mathrm{TBI}^{16}$. Evans Blue leakage analysis revealed minimal and transient vascular leakage at D3 and almost no vascular leakage thereafter in the dura mater (Supplementary Fig. 2a, d). In contrast, injured BVs of the pia mater and brain showed profound vascular leakage, particularly within the injury core, at D3 and D7 but sharply decreased over time. Nevertheless, this vascular leakage persisted at D28 (Supplementary Fig. 2b-d). Not only do these findings underscore the detrimental consequences of TBI in terms of brain edema, but they also highlight the superior regeneration potential of dura mater vascular integrity, in comparison to those in the pia mater and brain, after PTI.

VEGFR2, Tie2, and Dll4 contribute to vascular regeneration. As the dura mater showed complete vascular regeneration following PTI, whereas the brain contrastingly showed poor vascular recovery, further molecular characterization of these processes was performed in the dura mater and brain. VEGF-A/ VEGFR2 signaling has critical roles in angiogenesis and vascular regeneration, whereas VEGF-C/VEGFR3 and angiopoietin-Tie2 signaling play modulatory and facilitating roles in these biological processes ${ }^{17,18}$. Notch signaling, on the other hand, provides counterbalance to these driving forces via Dll4 in certain settings ${ }^{19,20}$. In the normal state, VEGFR2 and Tie2 could be readily detected in both the dura mater and brain BVs (Supplementary Fig. 3a-c, g), whereas Ang2 and Dll4 were hardly detected in either tissue (Supplementary Fig. 3d, e, h, i). At D3 however, when active sprouting angiogenesis occurs, VEGFR2, Ang2, and Dll4 were highly increased, whereas Tie2 was drastically reduced and no VEGFR3, which is restricted to lymphatic vessels, was detected in the ECs of BVs within and surrounding the injury areas of the dura mater (Fig. 2a, Supplementary Fig. 3c-f). In the injured brain, all molecules, excluding VEGFR3, were upregulated in the BVs within and surrounding the injury area (Fig. 2a, Supplementary Fig. 3g-i).

To assess the functional roles of VEGFR2, Tie2, and Notch signaling in vascular regeneration, we deleted VEGFR2, Tie2, or Dll4 in ECs in a tamoxifen-dependent manner using VEG$F R 2^{\mathrm{i} \triangle \mathrm{EC}}, T i e 2^{\mathrm{i} \triangle \mathrm{EC}}$, or Dll4 ${ }^{\mathrm{i} \triangle \mathrm{EC}}$ mutants, which were generated by crossing VE-cadherin-Cre-ER ${ }^{\mathrm{T} 2}$ mice $^{21}$ with VEGFR $2^{f l / f l}$ mice $^{22}$, Tie2 flfll mice ${ }^{23}$, or Dll4flfl mice ${ }^{24}$ (Fig. 2b). Cre-ER ${ }^{\mathrm{T} 2}$-negative but flox/flox-positive littermates were defined as wild-type control mice (control) in each experiment. D7 was chosen as the analyses timepoint, given that dura mater vascular density regenerates to pre-injury levels by 7 days after PTI. The vascular regeneration in the dura mater was drastically compromised by $88 \%$ in VEGFR $2^{\mathrm{i} \triangle \mathrm{EC}}$ mice, whereas it was reduced by $44 \%$ in $T i e 2^{\mathrm{i} \triangle \mathrm{EC}}$ mice (Fig. 2c-f) at D7. However, a longer period of healing allowed complete vascular regeneration in $\mathrm{Tie} \mathrm{2}^{\mathrm{i} \Delta \mathrm{EC}}$ mice, whereas VEGFR $2^{i \triangle E C}$ mice showed $91 \%$ reduction in vascular density in the injured dura mater at D14 (Supplementary Fig. 4a, b), indicating that VEGFR2 signaling is the predominant driver, whereas Tie2 signaling supports vascular regeneration only at the initial stage. Excessive angiogenesis with $43 \%$ increase in vascular density occurred with Dll4 deletion (Fig. 2e, f), implicating the critical role of Notch signaling in terminating angiogenesis when dura mater vascular regeneration is complete. Vascular density in the injured brain was only affected in the VEGFR2 $2^{\mathrm{i} \triangle \mathrm{EC}}$ mice, in which 59\% decrease was observed (Fig. 2g-j). To confirm the critical dependency of vascular regeneration on VEGFR2 signaling, VEGFR2 blocking antibody DC 101 ( $40 \mathrm{mg} / \mathrm{kg}$ of body weight) or control antibody IgG-Fc ( $40 \mathrm{mg} / \mathrm{kg}$ of body weight) was administered after PTI. Revascularization of avascular areas in the dura mater and brain was markedly suppressed by 74 and $56 \%$ with DC 101 treatment 


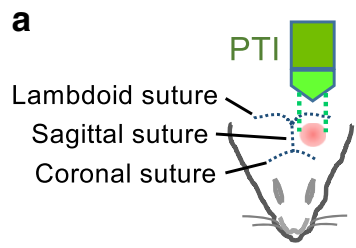

b 7 PTI or Sham

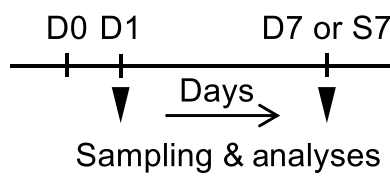

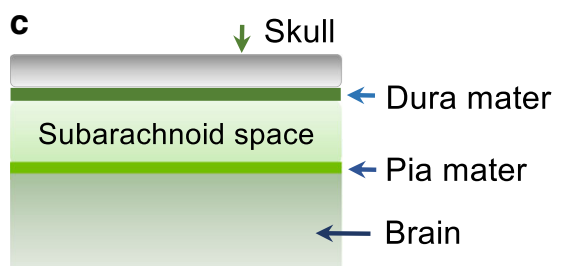

D7
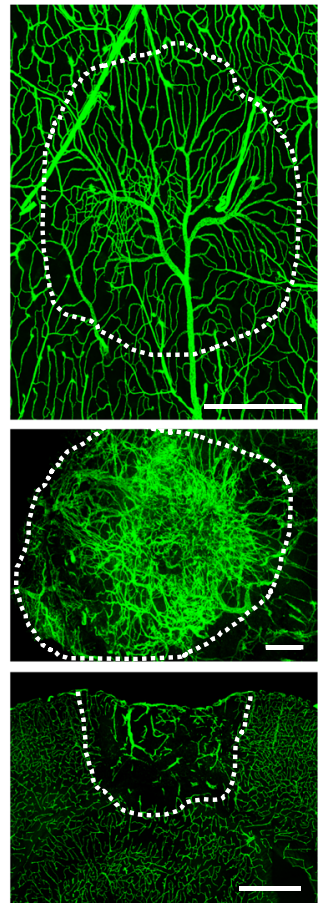

CD31 e

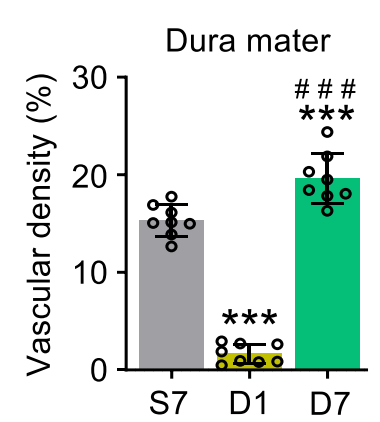

S7

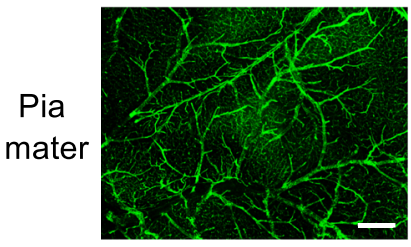

Brain

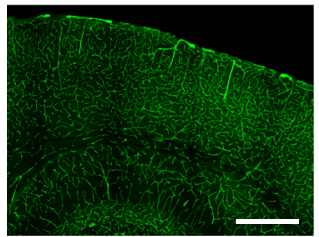

CD31

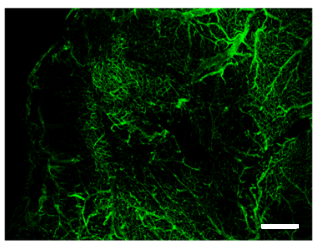

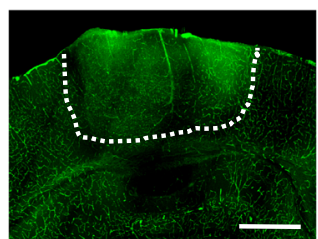

CD31
D1

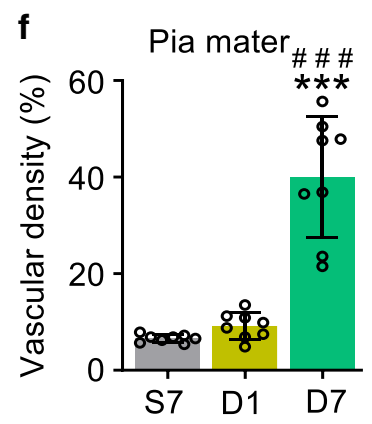

g

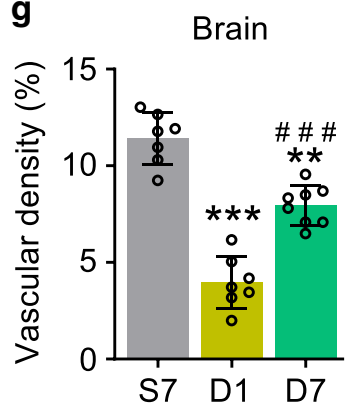

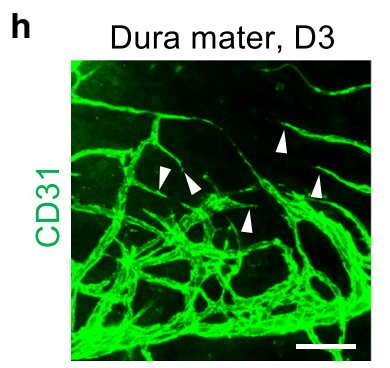

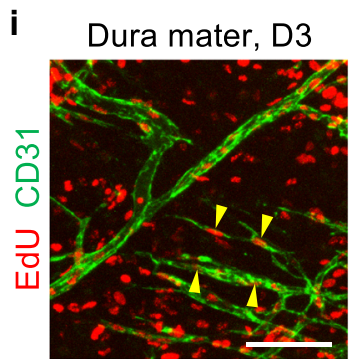

Pia mater, D3

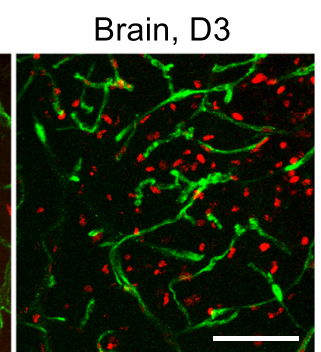

(Supplementary Fig. 5a-e), confirming that VEGFR2 signaling is critical for complete vascular regeneration in the injured dura mater. VEGFR2 signaling inhibition also suppressed vascular leakage in the brain by $44 \%$ with DC 101 treatment.

High VEGFR2 was well-coupled with pVEGFR2 and pERK in both tissues (Fig. 3a, b, Supplementary Fig. 6a, b) at D3, indicating that vascular regeneration is mainly mediated through activation of VEGFR2-ERK signaling. However, pAkt coupling was far more pronounced in the recovering vessels of the dura mater (Fig. 3c), compared with those of the brain (Supplementary Fig. 6c), and may confer angiogenic advantage to the former. Further temporal analyses of these phosphorylated molecules in 
Fig. 1 Differential vascular regeneration upon PTI in meninges and brain. a Schematic diagram depicting PTI onto mouse head as HI model. Pink circle indicates Rose Bengal activated by white light $(3 \mathrm{~mW})$. b Diagram depicting the experimental scheme for PTI or sham (Sham, S) operation, sampling, and analyses at indicated time points; D0, D1, and D7, day 0, 1, and 7 after PTI; S7, day 7 after sham operation. c Schematic diagram depicting layers of meninges between skull and brain. d Representative images of CD31 + BVs in dura mater, pia mater, and brain at S7, D1, and D7 in adult mice. Each white dotted-lined circle or line indicates putative injury area. Note rapid and organized vascular regeneration in the dura mater, whereas there is excessive or insufficient and disorganized vascular regeneration in the pia mater or brain. Scale bars, $500 \mu \mathrm{m}$. e-g Comparisons of CD31+ vascular densities in the indicated tissues. Each dot indicates a value from one mouse and $n=7$ (Brain S7, Brain D1), $n=8$ (all other groups) mice/group from three independent experiments. Vertical bars indicate mean \pm SD. ${ }^{\star \star \star} P<0.001$ versus S7 and ${ }^{\# \# \# ~} P<0.001$ versus D1 by Kruskal-Wallis test. $\mathbf{h}, \mathbf{i}$ Representative images of sprouting (white arrowheads) angiogenesis and EdU-incorporated EC proliferation (yellow arrowheads) at D3 in the indicated tissues of adult mice. Similar findings were observed in two independent experiments using $n=3-4$ mice. Scale bars, $100 \mu \mathrm{m}$.

the injury area of the dura mater showed pVEGFR2 and pAkt levels returning to basal levels by D7, and pERK by D14, all of which corresponded with the accompanying decrease in VEGFR2 (Fig. 3d-f). Significant increase of VE-PTP, an endotheliumspecific vascular endothelial-phosphotyrosine phosphatase $\mathrm{e}^{25}$, in regenerating vessels of both the dura mater and brain at D3 (Supplementary Fig. 7a-d) suggests that VE-PTP may be involved in such downstream regulation of VEGFR2 ${ }^{26}$.

Importantly, immunofluorescence analysis of human dura mater and brain cortex autopsy samples obtained from $\mathrm{HI}$ patients also showed substantial increase of VEGFR2 in both the dura mater and brain BVs within the injury area when compared with the contralateral side (Fig. 4a-d), underscoring the clinical relevance of increased VEGFR2 in HI.

Therefore, these results implicate key molecular pathways governing vascular regeneration in the dura mater following PTI, with VEGFR2 signaling playing a predominant and critical role.

PDGFR $\beta+$ cells stabilize vascular regeneration after injury. Pericytes or perivascular cells play diverse roles in angiogenesis, vascular remodeling, regeneration and stabilization ${ }^{27}$. We labeled PDGFR $\beta+$ cells using a PDGFR $\beta$-Cre-ER ${ }^{\mathrm{T} 2}$-tdTomato reporter mouse model (Supplementary Fig. 8a). Although most PDGFR $\beta+$ cells are tightly associated with CD31+ brain capillaries as pericytes, they are spatially dichotomous in the dura mater, where they are either associated with dural BVs or randomly distributed in the stroma like fibroblasts (Supplementary Fig. 8b). Disorganized accumulation of PDGFR $\beta+$ cells surrounding angiogenic vessels occurred in both the dura mater and brain at D3 (Supplementary Fig. 8c). To address the role of PDGFR $\beta+$ cells in vascular regeneration, PDGFR $\beta$-blocking antibody $\mathrm{APB}^{28}$ or control antibody IgG-Fc was administered after PTI. Although there was no notable difference in the vascular density of the recovered area, the diameter of the recovered vessels was increased by 2.3 -fold in the dura mater and brain with APB treatment compared with those with IgG-Fc treatment (Supplementary Fig. 8d, e). Further characterization of NG2+ perivascular cells after APB5 treatment showed significantly decreased NG2 + cell number, as well as poor perivascular coverage by these cells (Supplementary Fig. 8f, g). Thus, PDGFR $\beta+$ cells play a stabilizing role in the overall structure of the regenerative BVs in the dura mater and brain upon injury.

Diverse macrophage behaviors in meninges and brain after PTI. It has recently been demonstrated that F4/80+, LYVE1+, and CD206+ macrophages are adjacently aligned with capillaries of the dura mater, and play surveillance roles as the secondary interface between the systemic circulation and the $\mathrm{CNS}^{10,29}$. As LYVE1+ macrophages have been reported to contribute to angiogenesis and $\mathrm{BV}$ homeostasis in various tissues ${ }^{30-32}$, we examined the spatiotemporal distribution of LYVE1+ macrophages after PTI. Distribution of LYVE1+/F4/80+ macrophages was markedly reduced in the dura mater injury core during vascular regeneration, but they were highly distributed with activated shapes at the injury margin at D3 (Supplementary Fig. 9a-c). In contrast, single F4/80+ macrophages were profoundly accumulated within the injury core with activated shapes at D3 (Supplementary Fig. 9a-c). Beneath the dura mater, distribution of LYVE1+/F4/80+ macrophages was confined to the pia mater, whereas single $\mathrm{F} 4 / 80+$ macrophages were heavily accumulated in the injury core of brain at D3 and D7 (Supplementary Fig. 9d, e). Given that residential dura mater macrophages and brain microglia are distinct from bone marrowderived circulating macrophages, we investigated the origin of the activated macrophages in the injury area following PTI. Analyses of WT:actin-GFP parabiosis recipient mice showed complete absence of GFP-expressing macrophages in the injured dura mater, whereas a significant proportion of macrophages in the injured brain expressed GFP (Supplementary Fig. 9f-h). These results indicate that activation and accumulation of dura mater macrophages occur internally, whereas these processes occur in the brain with substantial contribution from circulating monocytes/macrophages.

Macrophages play minor role in dural vascular regeneration. Activated macrophages are known to be major suppliers of VEGF-A in the context of tissue injury ${ }^{33,34}$. To clarify the predominant source of VEGF-A during vascular regeneration, we examined the expression and production of VEGF-A using a VEGF-LacZ reporter mouse model. VEGF-A was not upregulated in both LYVE1+/F4/80+ and single F4/80+ macrophages in the dura mater at D3 (Fig. 5a). In comparison, VEGF-A was highly upregulated in single $\mathrm{F} 4 / 80+$ macrophages in the injury core of brain and in LYVE1+/F4/80+ macrophages in the pia mater at D3 (Fig. 5a). VEGF-A expression was predominantly high in Osterix + osteoprogenitors/osteoblasts in the calvarial bone (Fig. 5a, b) and in the normal portions of neurons in the brain (Fig. 5a). RT-PCR analysis of the dura mater revealed no significant differences of mRNA expression of $V e g f a, V e g f c$, and $P g f$ between sham-operated day 3 (S3) and D3 (Fig. 5c). Moreover, enzyme-linked immunosorbent assay (ELISA) revealed no significant difference of VEGF- $\mathrm{A}_{164}$ in the dura mater and brain between S3 and D3 (Fig. 5d). Single-cell RNAseq analyses of fluorescence-activated cell sorting (FACS)-sorted CD45+ hematopoietic cells (Supplementary Fig. 10a, b) showed sparse Vegfa expression in various immune cells, such as in granulocytes, mast cells, innate lymphoid cells, and eosinophils of the dura mater and brain in the sham condition (Fig. 5e). Vegfa was largely absent in macrophages of both tissues and in brain-resident microglia populations. Upon injury, a new macrophage population expressing strong Vegfa levels emerged within the injured brain, whereas no Vegfa expression changes could be detected within hematopoietic cells of the dura mater following PTI (Fig. 5e, f).

Finally, to evaluate the potential contribution of these macrophages to vascular regeneration, we genetically depleted 
a
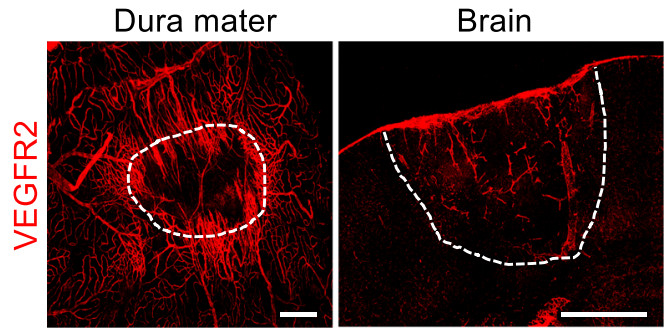

C
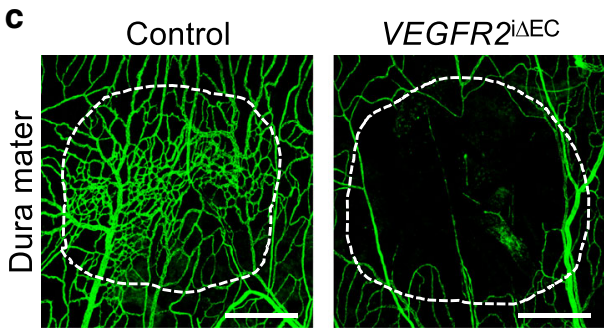

d

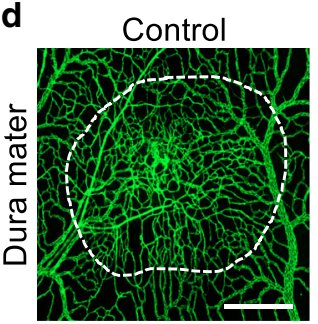

Tie2 $^{\mathrm{i} \Lambda \mathrm{EC}}$

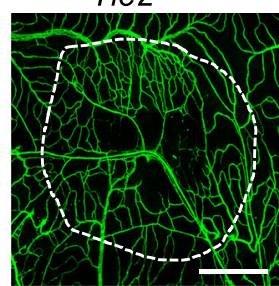

e

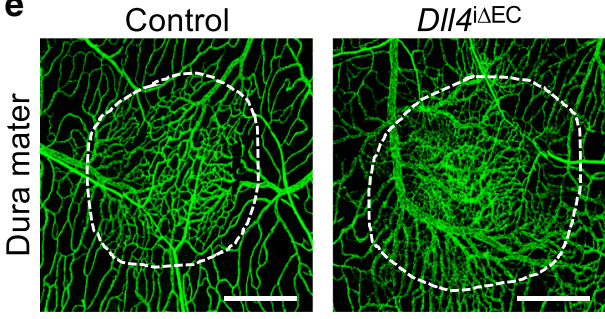

CD31 b VEGFR2 $2^{\mathrm{i} \triangle \mathrm{EC}}, T i e 2^{\mathrm{i} \Delta \mathrm{EC}}$ or $D / 14^{\mathrm{i} \Delta \mathrm{EC}}$ mice

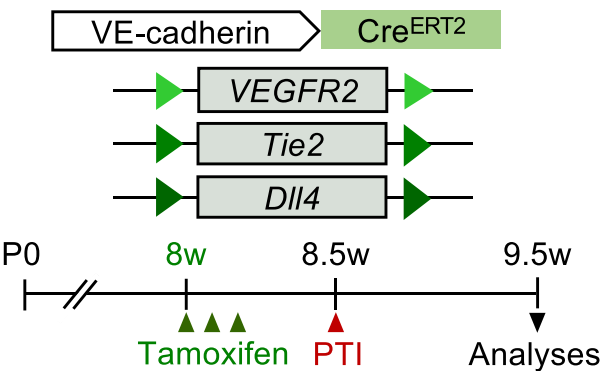

Analyses

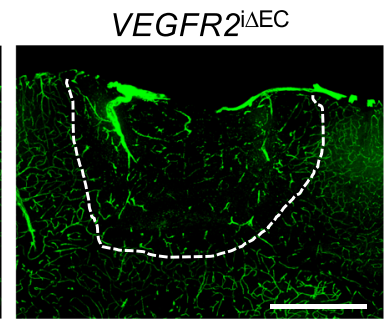

$\mathbf{h}$
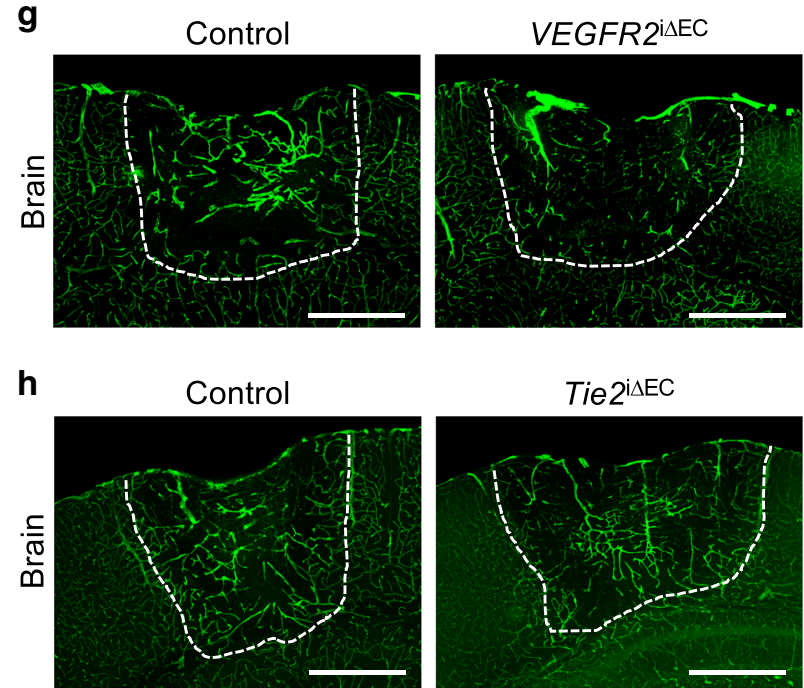

i
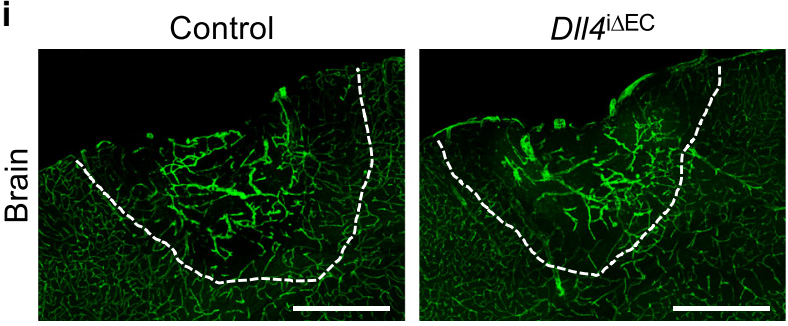

CD31

\section{Control Control Control \\ VEGFR2 ${ }^{\mathrm{i} E \mathrm{EC}} \square$ Tie2 $2^{\mathrm{i} E \mathrm{EC}} \square \mathrm{DI} 4^{\mathrm{i}} \mathrm{EC}$}

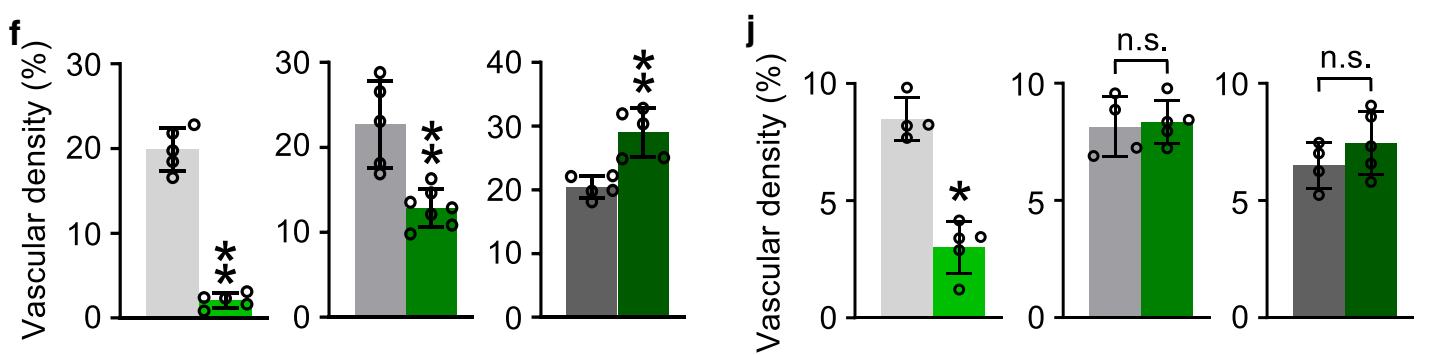

Fig. 2 VEGFR2 signaling is critical for vascular regeneration in dura mater and brain after PTI. a Representative images of VEGFR2 upregulation in the surrounding BVs of putative injury areas (white dotted-lined circle or line) at D3 in adult mice. Similar findings were observed in four mice from two independent experiments. Scale bars, $500 \mu \mathrm{m}$. b Diagram depicting generation of VEGFR2 $2^{\mathrm{i} E \mathrm{EC}}$, Tie $2^{\mathrm{i} \Delta \mathrm{EC}}$, or D/l4 ${ }^{\mathrm{i} \Delta \mathrm{EC}}$ mice and EC-specific deletion of VEGFR2, Tie2, or DI/4 in 8-week-old mice by i.p. injections of tamoxifen, PTI, and their analyses at 1 week after PTI. c-j Representative images and comparisons of CD31+BVs of putative injury areas (white dotted-lined circle or line) in the dura mater and brain at D7 in control and VEGFR2 ${ }^{\mathrm{i}} \mathrm{EC}, \mathrm{Tie}^{\mathrm{i}} \triangle \mathrm{EC}$

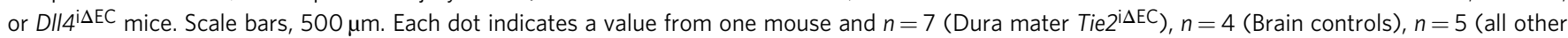
groups) mice/group from two independent experiments. Vertical bars indicate mean $\pm \mathrm{SD}$. ${ }^{\star} P<0.05$, ${ }^{\star \star} P<0.01$ versus control by two-tailed Mann-Whitney $U$ test. 
a

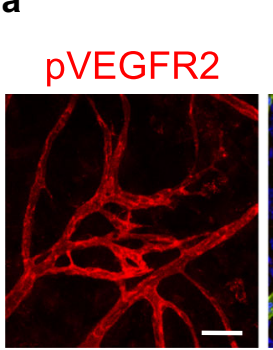

b
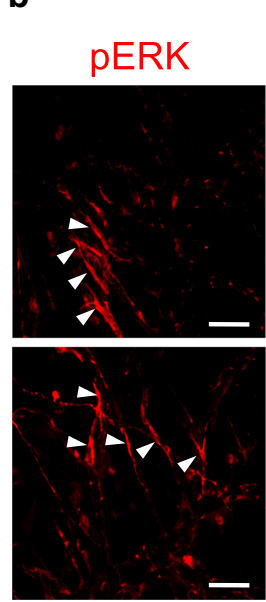

C

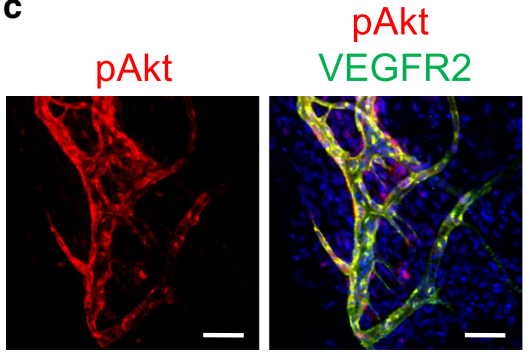

pVEGFR2

VEGFR2
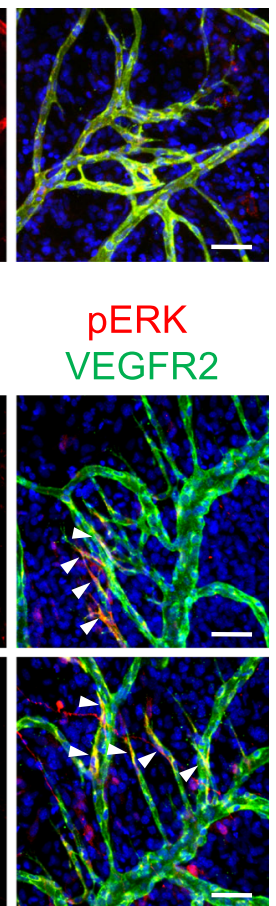

d
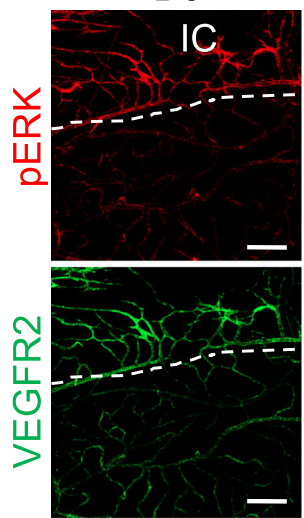

$\mathbf{f}$
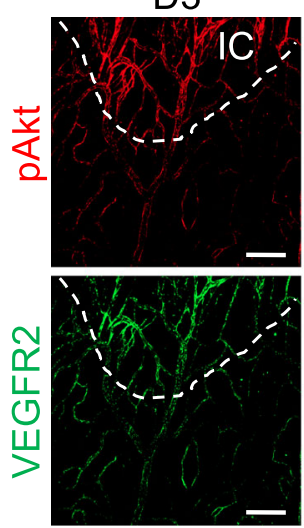

D7
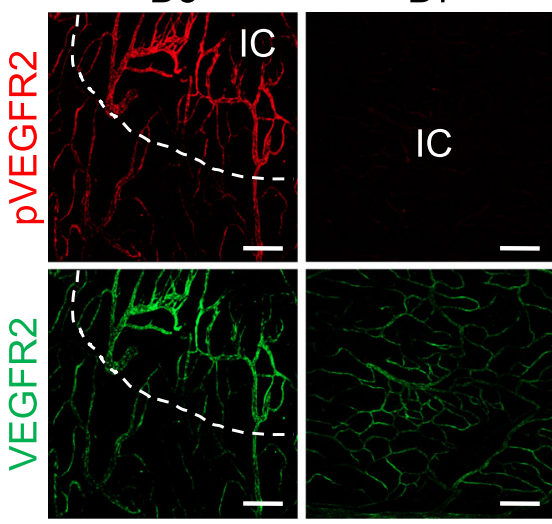

D7

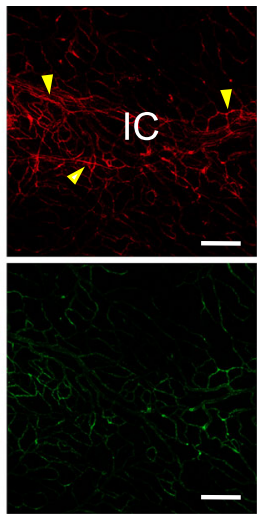

D7
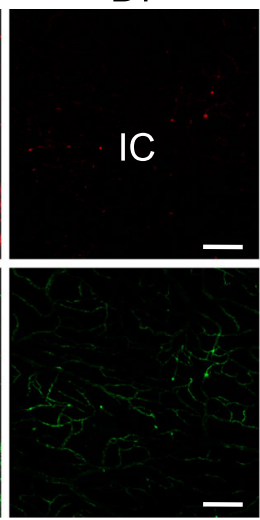

D14
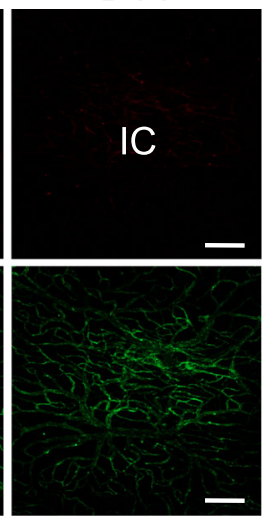

D14

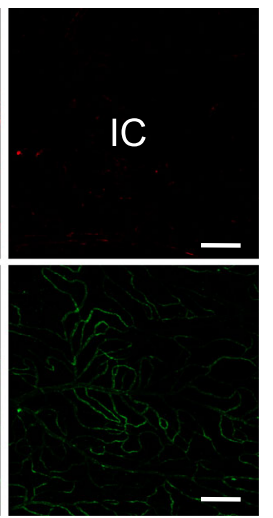

D14
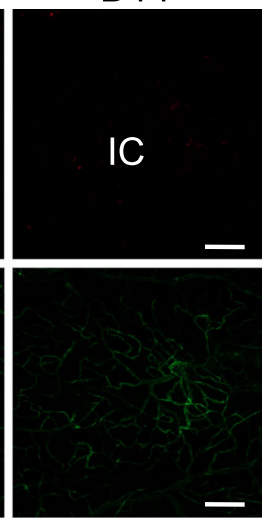

Fig. 3 Spatiotemporal changes of pVEGFR2, pERK, and pAkt in VEGFR2 + blood vessels during vascular regeneration in dura mater after PTI. a-c Representative images of phosphorylated VEGFR2, ERK (white arrowheads), and Akt in regenerating BVs of the dura mater at D3 after PTI in adult mice. Scale bars, $50 \mu \mathrm{m}$. Similar findings were observed in four mice from two independent experiments. $\mathbf{d}$-f Representative images of $\mathrm{pVEGFR2,} \mathrm{pERK}$, and $\mathrm{pAkt}$ in VEGFR2+BVs of surrounding BVs of putative injury area (white dotted line) or injury core (IC) at D3, D7, and D14 after PTI in adult mice. Similar findings were observed in $n=3$ mice from two independent experiments. Scale bars, $200 \mu \mathrm{m}$. Note that pERK remains elevated at D7 (yellow arrowheads) before returning to basal levels by D14.

monocytes/macrophages using a $C \times 3 c r 1^{\text {DTR }}$ mutant mouse model. Systemic administration of diphtheria toxin ablated macrophages in most tissues by $85-98 \%$ and caused $64 \%$ and $46 \%$ reductions, respectively, in vascular regeneration in the injured pia mater and brain, respectively (Supplementary Fig. 11a-f), but impact on dura mater vascular regeneration was minimal (Fig. 5g, h). These data indicate that infiltrating macrophages expressing high VEGF-A in the pia mater and brain are required for vascular regeneration, whereas accumulated macrophages in the dura mater play minor facilitating roles in vascular regeneration.

Inflammatory role of dura mater macrophages following PTI. As high VEGF-A expression was found only in activated macrophages of the injured brain (Supplementary Fig. 12a), we 
a
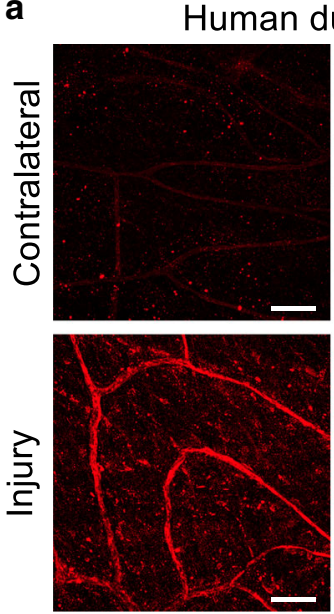

VEGFR2
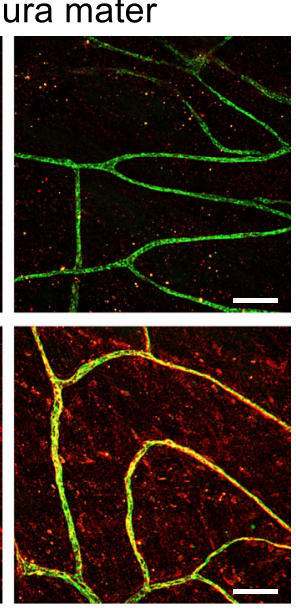

VEGFR2 CD31
C
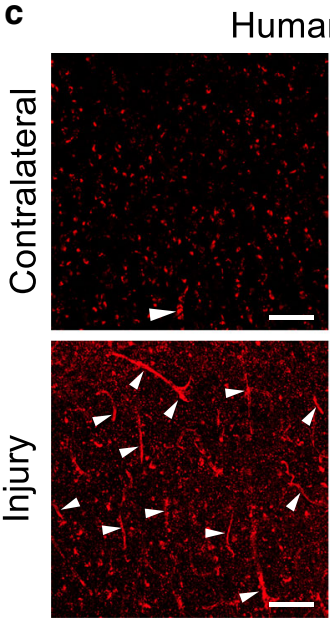

VEGFR2
Human brain
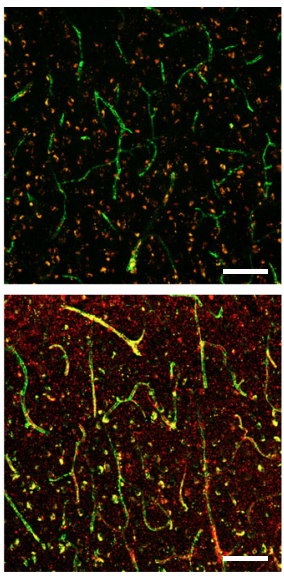

b

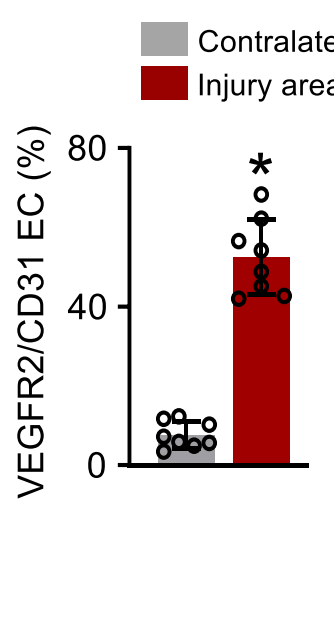

d
Contralateral side Injury area

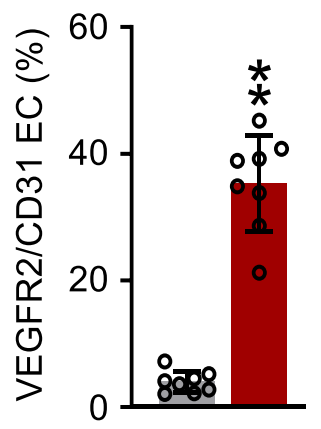

Fig. 4 VEGFR2 upregulation in blood vessels of injured dura mater and brain in human patients with traumatic head injury. a-d Representative images and comparisons of VEGFR2 upregulation in the BVs of injury area and contralateral (CL) side of human dura mater and brain at $24 \mathrm{~h}$ post-mortem from patients with HI. White arrowheads indicate VEGFR2 + BVs. Scale bars, $100 \mu \mathrm{m}$. Two values were obtained from two areas of each patient sample, $n=4$ patients from four independent experiments. Vertical bars indicate mean $\pm \mathrm{SD}$. ${ }^{\star \star} P=0.0002$ versus $\mathrm{CL}$ by two-tailed Mann-Whitney $U$ test.

sought to explore other angiogenic factors expressed by dura mater macrophages. Single-cell RNAseq analyses of CD45+ hematopoietic cells found in the dura mater and brain showed a distinct Lyve1+ sub-cluster of macrophages (Supplementary Fig. 10a, b). Further comparative analyses between Lyve1+ and Lyve 1- macrophages within the dura mater revealed that the expression of multiple MHC class II molecules was enriched in the latter (Supplementary Fig. 12b), suggesting the specialized immune-surveillance role of these cells in dura mater protection against infection. Following injury, these dura mater-resident Adgre 1+ macrophages showed elevated expression of proinflammatory molecules, such as $\mathrm{Ccl} 2, \mathrm{Ccl} 7, \mathrm{Ccl} 8, \mathrm{Ccl} 9$, and Cxcl2 (Supplementary Fig. 12d). Significant changes in proangiogenic molecules could not be detected in the Lyve1+ or Lyve 1-macrophages of the injured dura mater. Brain-infiltrating macrophages, on the other hand, had increased expression of a wide range of angiogenic and anti-inflammatory molecules, such as Vegfa, Lgals1 \& 3, and Iqgap1 (Supplementary Fig. 12c, e). Interestingly, anti-angiogenic molecules, such as Thbs1, Runx3, Tmsb10, Tgfbi, and Pgk1, were also upregulated, suggesting another potential explanation for poor vascular regeneration in the injured brain. Therefore, these results confirm the absence of increased angiogenic contribution from dura mater macrophages, which are quite unique in molecular nature, or from any other hematopoietic cell within the injured dura mater.

VEGFR2 signaling is required for dural vascular homeostasis. Our observation of robust VEGFR2 and Tie2 expression in dural BVs led us to explore the roles of VEGFR2 and Tie2 signaling in adult vascular homeostasis by examining the phenotypes of BVs and perivascular macrophages in the VEGFR2 $2^{\mathrm{i} \triangle \mathrm{EC}}$ and Tie $2^{\mathrm{i} \Delta \mathrm{EC}}$ mice (Fig. 6a). Compared with those of control mice, diameters of capillaries and venules were $\sim 50 \%$ thinner and vascular density was reduced by $47 \%$ in $V E G F R 2^{\mathrm{i} \triangle \mathrm{EC}}$ mice but remained similar in Tie $2^{\mathrm{i} \Delta \mathrm{EC}}$ mice (Fig. 6b, c). Nevertheless, no differences were found in vascular density of the brain, and distribution, alignment and density of LYVE1+/F4/80+ and single F4/80+ perivascular macrophages among control, VEGFR2 $2^{\mathrm{i} \triangle \mathrm{EC}}$, and Tie $2^{\mathrm{i} \Delta \mathrm{EC}}$ mice (Fig. 6b-e). These findings indicate that VEGFR2, but not Tie2, signaling is required to maintain dural vascular homeostasis.

Dura mater ECs intrinsically possess angiogenic potential. To assess EC-intrinsic advantages to vascular regeneration, an 
a

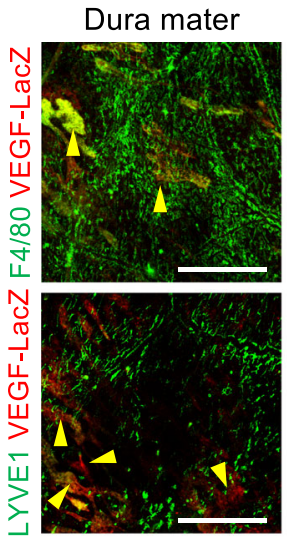

b

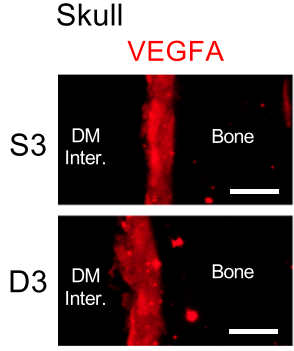

e

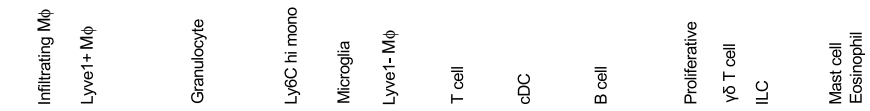

Sham

2
1 Level of
Vegfa
mRNA
-1

Injury

|

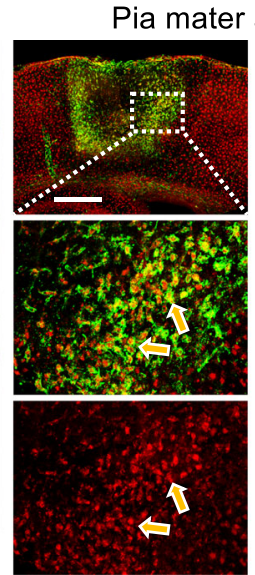

F4/80 VEGF-LacZ LYVE1 VEGF-LacZ
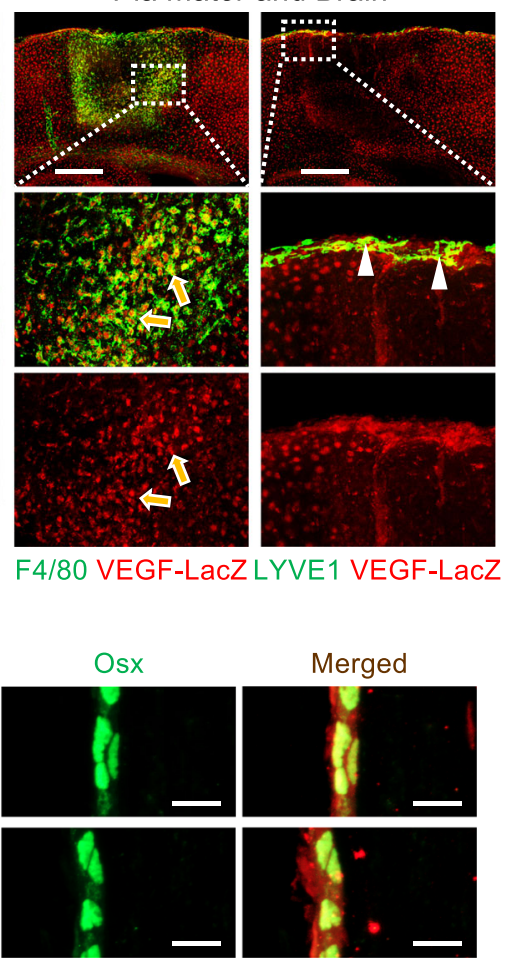

C

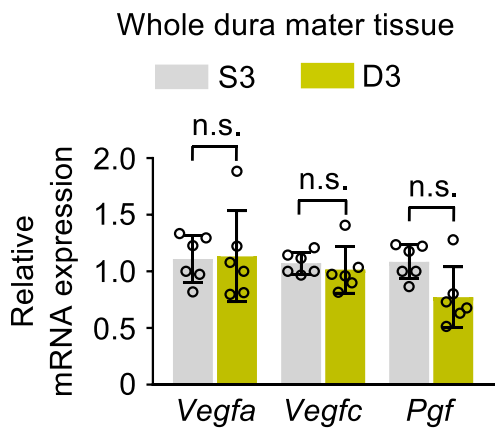

d

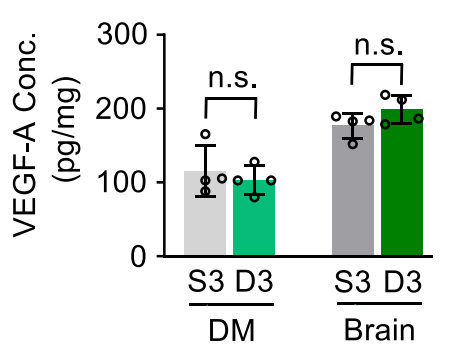

g

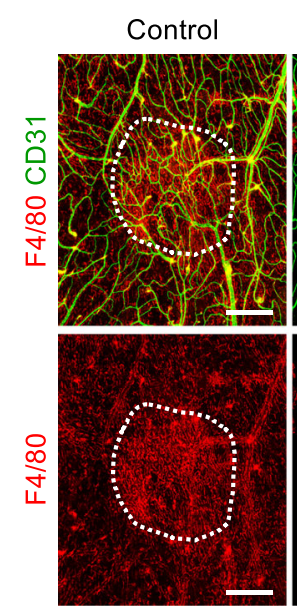

CX3-DTR (\#1)
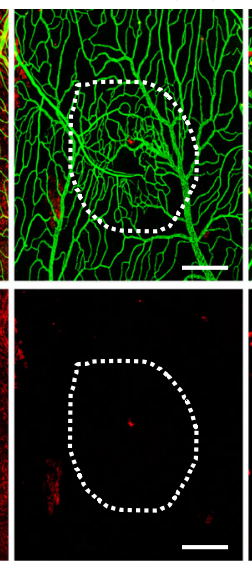

CX3-DTR (\#2)
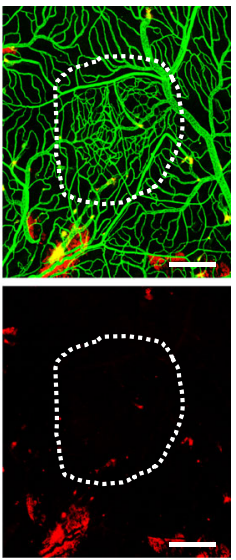

f Dura mater Brain

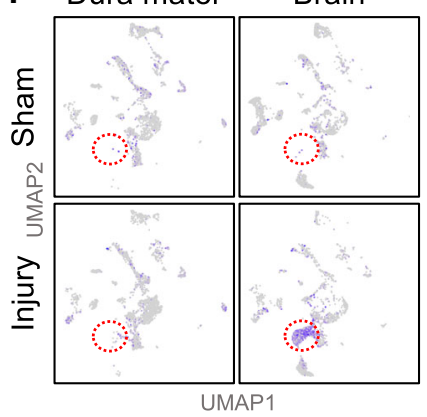

h

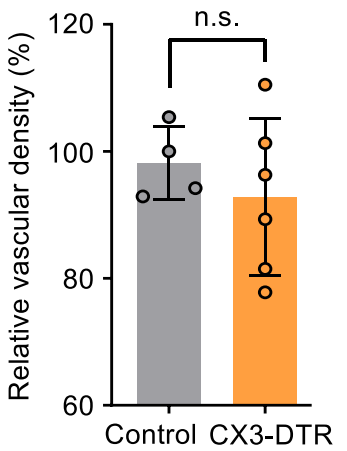

ex vivo sprouting assay ${ }^{35}$ was performed using FACS-sorted dura mater or brain ECs. Although robust sprouting and filopodia were observed in dural EC spheroids, almost no sprouting and filopodia were detected in brain EC spheroids (Fig. 7a-c), confirming that dura mater ECs have a more vigorous intrinsic angiogenic potential than brain ECs.
We then performed RNAseq analysis to further elucidate fundamental molecular differences between these tissue-specific ECs. A majority of 1020 genes from 1343 differentially expressed genes (DEGs) between dura mater and brain ECs were upregulated in the former (Fig. 7d). Ingenuity Pathway Analysis (IPA) analysis of these changes showed strong enrichment of 
Fig. 5 Dura mater macrophages play minor role in meningeal vascular regeneration. a Representative images of VEGF-A expression by detection of $\beta$-galactosidase activity in VEGF-LacZ mice at D3. High expression of VEGF-A is detected in neighboring calvarial bone (yellow arrowheads), F4/80+ macrophages (yellow arrows) in brain, and LYVE1+ macrophages (white arrowheads) in pia mater. Similar findings were observed in $n=3$ mice from two independent experiments. Scale bars, $500 \mu \mathrm{m}$. b Representative images of VEGF-A in Osterix $+(\mathrm{Osx}+)$ osteogenic progenitor cells/osteoblasts along the endosteal surface of calvarial bone of adult mice at D3 after PTI. No difference in VEGF-A between S3 and D3 is shown. Similar findings were observed in $n_{-}=3$ mice from two independent experiments. DM Inter., dura mater interface. Scale bars, $10 \mu \mathrm{m}$. c, d Relative mRNA expressions of Vegfa, Vegfc, and Pgf in whole dura mater tissue and VEGF-A protein concentrations in the dura mater (DM) and brain at D3. Each dot indicates a value obtained from one mouse and $n=6 \mathbf{c}, n=4 \mathbf{d}$ mice/group from three independent experiments. Vertical bars indicate mean \pm SD. n.s., not significant versus each S3 by twotailed Mann-Whitney $U$ test. e Heatmap of Vegfa expression based on single-cell RNAseq analysis of CD45+ hematopoietic cells isolated from dura mater and brain at D3. Emergence of infiltrating macrophages with high Vegfa expression is observed after PTI. f Feature plots showing Vegfa expression in various CD45+ hematopoietic cell clusters at D3. Note emergence of infiltrating macrophages showing high Vegfa expression only in brain after PTI (red dotted circle). $\mathbf{g}$, h Representative images and comparisons of vascular regeneration in the dura mater between control mice and CX3CR1-DTR mice (two cases \#1 and \#2 are shown) at D7. White dotted-lined circles indicate putative injury areas. Scale bars, $500 \mu$ m. Each dot indicates a mean value obtained from one mouse and $n=4$ (S3), $n=6$ (D3) mice/group from two independent experiments. Vertical bars indicate mean \pm SD. n.s., not significant versus control by two-tailed Mann-Whitney $U$ test.

vascular development and angiogenesis pathways (Fig. 7e, Supplementary Fig. 13a, b). Interestingly, integrin signaling and integrin-linked kinase signaling showed high enrichment in the dura mater ECs (Supplementary Fig. 13c), suggesting that certain molecules within these pathways may contribute to the angiogenic state of the dura mater by cooperating with VEGFR2 as previously described ${ }^{17}$. siRNA-mediated knockdown of several candidates found highly enriched in dura mater ECs (Aqp1, Fabp4, and Esm1) significantly impaired sprouting angiogenesis by $65 \%, 84 \%$, and $76 \%$, respectively (Fig. $7 f$, g).

Complete regeneration of dura mater vessel basement membrane. We further characterized vascular responses to PTI in the dura mater and brain to identify additional tissue-specific differences. Of note, collagen IV+ basement membrane (BM) along the dural vessels was relatively well preserved, similar to tumor vessel regression ${ }^{36}$, following injury, whereas CD31+ECs were markedly damaged at D1 (Fig. 8a, b). At D14, by which vascular function is fully restored to pre-injury conditions, the alignment of collagen IV $+\mathrm{BM}$ and shape of the regenerated BVs fully recovered, looking like pre-injury normal BVs, in the dura mater (Fig. 8a, b). In contrast, collagen IV along the brain vessels was highly increased at D1 and continued to accumulate, irrespective of vascular patterning, within the injury core until D14 (Fig. 8c, d), highlighting the superior functional regeneration, with extrinsic BM support, of dura mater BVs following PTI.

Vascular regeneration is required for dural tissue recovery. Considering the role of vascular regeneration in dura mater function, we investigated the consequences of incomplete vascular regeneration in the dura mater. Vascular regeneration inhibition with prolonged DC101 treatment for 2 weeks following PTI showed a substantial reduction in dura mater-resident F4/80+ macrophages in the avascular area (Fig. 9a-d). We also examined the extent of fibrosis within the avascular area using the fibroblast marker vimentin. After prolonged inhibition of vascular regeneration following PTI in EC-specific VEGFR2 knockout mice, density of vimentin + fibroblast was increased by $\sim 50 \%$ in VEGFR2 ${ }^{\mathrm{i} \triangle \mathrm{EC}}$ mice compared with control mice at D14 (Fig. 9e, f), implying that complete dura mater vascular regeneration is required to prevent excessive fibrosis at the injury site. Similarly, inhibition of VEGFR2-mediated vascular regeneration following an ear skin wound also led to the increase of fibrotic tissue surrounding the injury core at D14 (Supplementary Fig. 14a, b). However, no difference in the number of broken nerve fibers was observed at D14 in the dura mater between control and VEG$F R 2^{\mathrm{i} \triangle \mathrm{EC}}$ mice (Fig. 9g, h), suggesting that vascular regeneration and peripheral nerve regeneration are not coupled in this context.
Therefore, these results implicate efficient vascular regeneration as a critical mediator in the restoration of immune homeostasis and wound healing of the injured area following HI.

\section{Discussion}

Our study identifies key pathways of vascular regeneration in the dura mater following HI. We implicate VEGFR2 signaling as the predominant driver for dura mater vascular homeostasis and regeneration, with multiple intrinsic/extrinsic factors creating a primed state for rapid vascular regeneration.

Various impact injury models ${ }^{37-39}$ better simulate direct tissue damage from head trauma. However, the PTI model employed in this study is most useful for the side-by-side comparison of the meninges and the brain parenchyma following vascular injury, because it allows minimally invasive, deep-penetrating, consistent, and direct injury of BVs, with most tissue damage hallmarks associated with head trauma.

A previous study attributes dura mater vascular regeneration to macrophage-supplied MMP-2, which facilitates vascular regeneration by breaking down fibrin deposits accumulated within the injury core ${ }^{14}$. Our results do not show changes in VEGF-A levels but indicate that endothelial VEGFR2 signaling is upregulated, both in human HI patients and mice, and drives dura mater vascular regeneration. Unlike minimal suppression of vascular regeneration with macrophage depletion, we observed complete inhibition of vascular regeneration with EC-specific deletion or antibody blockade of VEGFR2. In contrast to low transcription levels, substantial amounts of VEGF-A protein in the dura mater suggest its supply from neighboring tissue, such as calvarial bone or neurons.

Although our study demonstrates that both the meninges and brain vessels rely on VEGFR2 for their differential vascular regeneration following PTI, molecular profiling of dura mater vs brain ECs based on RNAseq suggests that other specialized pathways may confer angiogenic and vascular regenerative advantages upon dura mater ECs and warrants further investigation. Also, how such dura mater EC-specific molecules contribute to suppressing vascular leakage, despite lacking a blood-brain barrier present in the brain ${ }^{9}$, remains to be explored.

Although the functional significance of meningeal lymphatics has recently received the spotlight ${ }^{40-42}$, the role of meningeal BVs has been rather unclear. Our study demonstrates the critical role dura mater BVs play in the functional regeneration of the dura mater tissue, with respect to its immunological function and stromal integrity. Although the dura mater vascular bed is separate from the pia mater and brain interconnected beds ${ }^{8,9}$ and therefore does not inflict direct vascular damage to the latter if injured, how the functional recovery of the dura mater potentially 
a

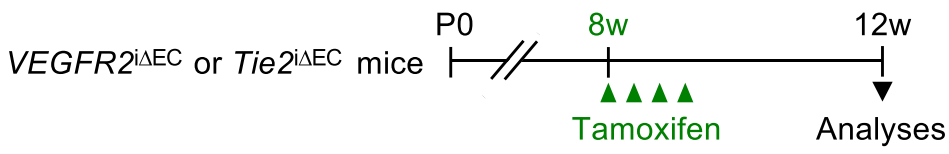

b
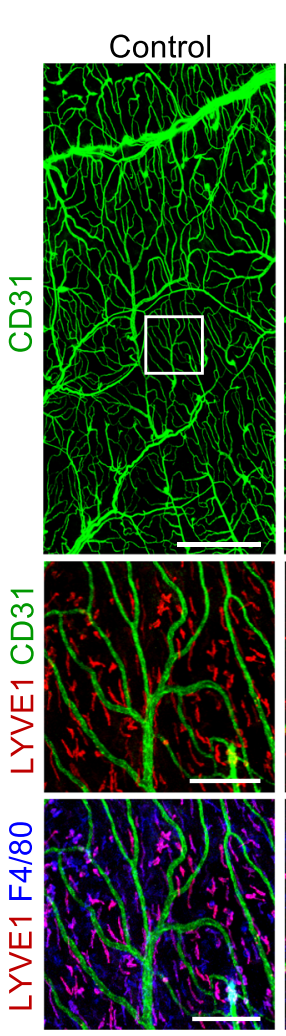

d

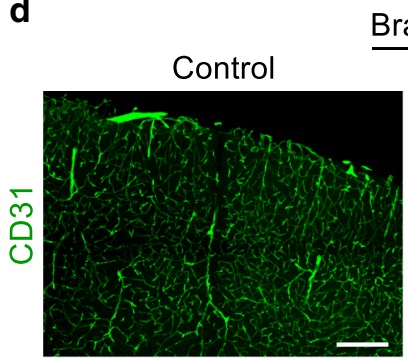

Control

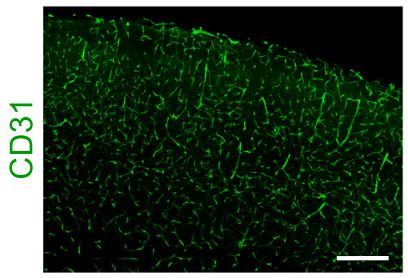

Dura mater
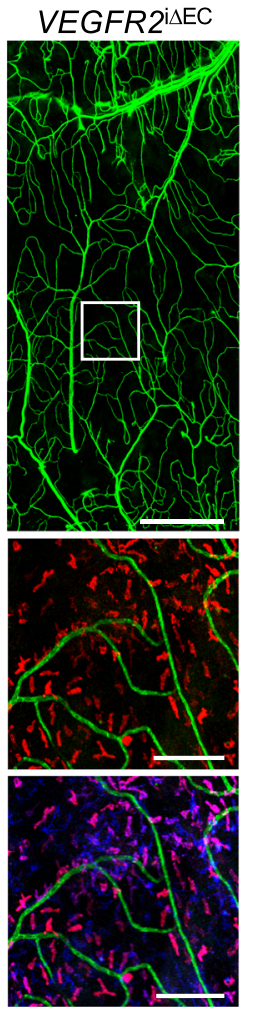

Brain

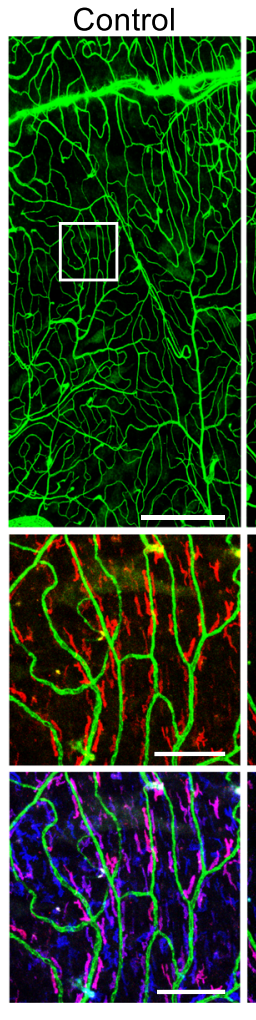

Tie2 ${ }^{\mathrm{i} \Delta \mathrm{EC}}$
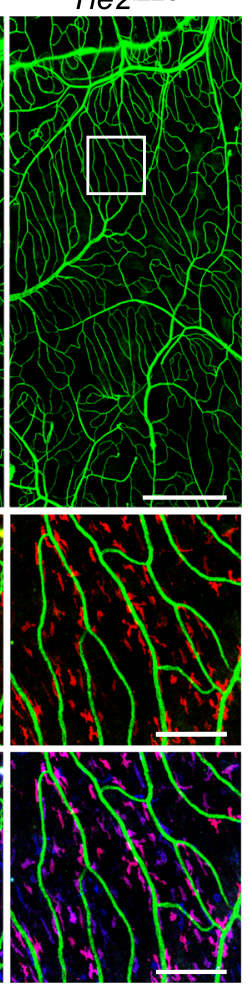

e

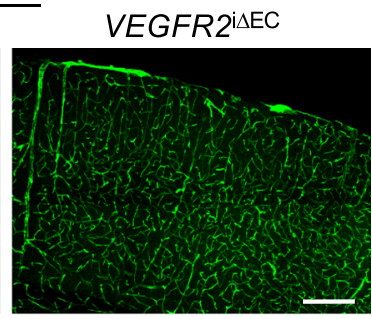

Tie2 ${ }^{\mathrm{i}} \Delta \mathrm{EC}$

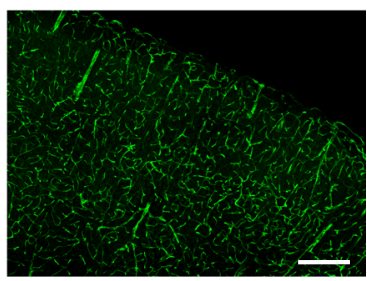

C
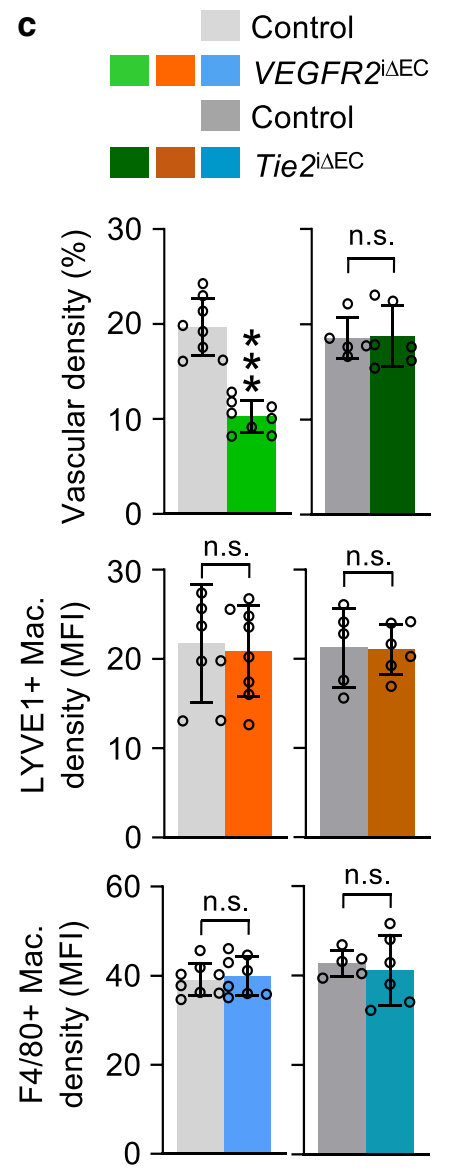

Fig. 6 VEGFR2, but not Tie2, signaling is required for maintaining dural vascular homeostasis. a Diagram depicting generation of VEGFR2 $2^{i} E \mathrm{EC}$ or $T i 2^{i} \triangle E C$ mice and EC-specific deletion of VEGFR2 or Tie2 at 8 weeks old of age and their analyses 4 weeks later. b Representative images of CD31+BVs (upper panels) and distributions of LYVE1+ (middle and lower panels) and F4/80+ macrophages (lower panels) in dura mater of control, VEGFR2 ${ }^{\mathrm{i} E \mathrm{EC}}$ and Tie ${ }^{\mathrm{i}} \Delta \mathrm{EC}$ mice. Scale bars, $1 \mathrm{~mm}$. Each white-lined box is magnified in middle and lower panels. Scale bars, $200 \mu \mathrm{m}$. c Comparisons of densities of CD31 $+B V s$, LYVE1+macrophages and F4/80+ macrophages in control and VEGFR2 ${ }^{i} \Delta E C$ or Tie2 ${ }^{i \Delta E C}$ mice. Each dot indicates a value from one mouse and $n=5$ (Tie2 control), $n=6$ (Tie2 ${ }^{i \Delta E C}$ ) $n=8$ (all other groups) mice/group from three independent experiments. Vertical bars indicate mean \pm SD. n.s., not significant or ${ }^{\star \star \star} P<0.001$ versus control by two-tailed Mann-Whitney $U$ test. MFI, mean fluorescence intensity. d, e Representative images of CD31+BVs in brain of control, VEGFR2 ${ }^{i \triangle E C}$, and Tie $2^{i \Delta E C}$ mice. Scale bars, $200 \mu \mathrm{m}$. Comparisons of vascular density between control and VEGFR2 ${ }^{i \Delta E C}$ or Tie2 $2^{i \Delta E C}$ mice. Each dot indicates a value from one mouse and $n=5$ (Tie2 control), $n=6$ (Tie ${ }^{i \Delta E C}$ ), $n=8$ (all other groups) mice/group from three independent experiments. Vertical bars indicate mean \pm SD. n.s., not significant versus control by two-tailed Mann-Whitney $U$ test. 
a
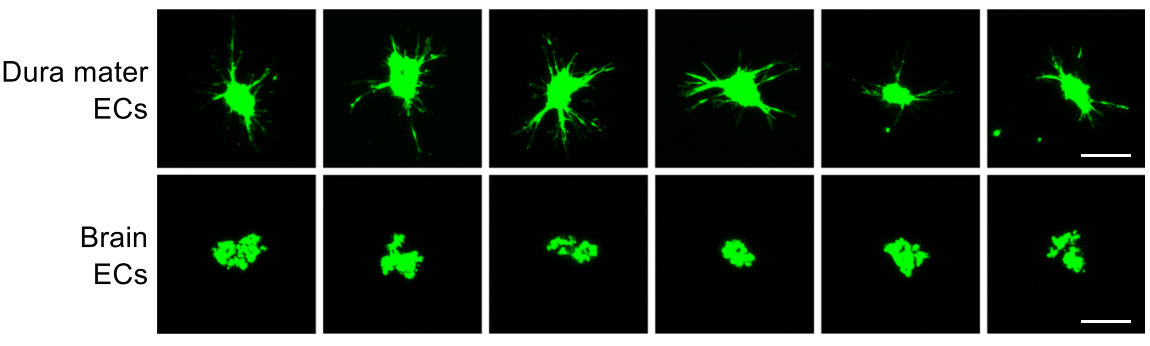

b

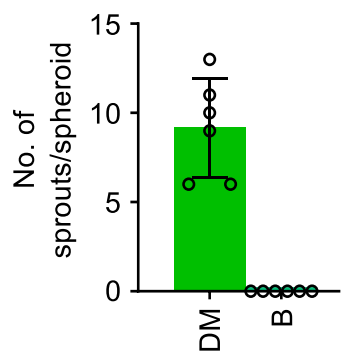

d

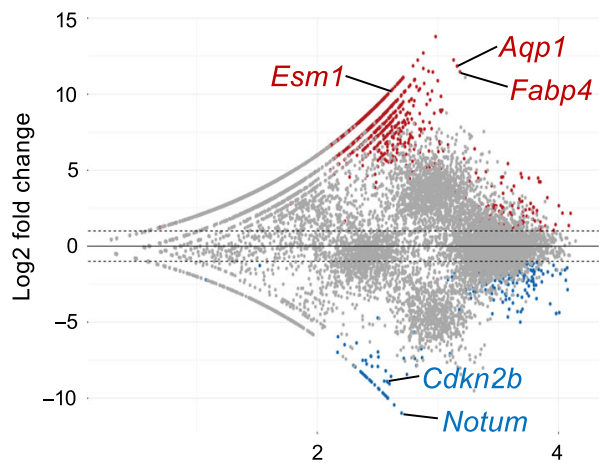

Log2 mean expression

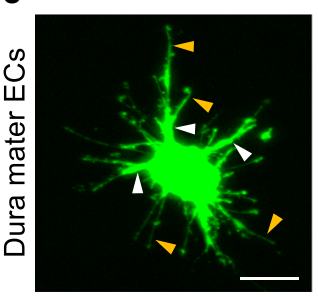

e

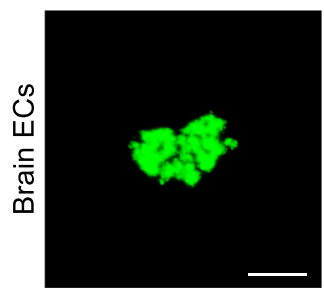

Activation Z-score

$\begin{array}{lllll}0 & 1 & 2 & 3 & 4\end{array}$

nt of vasculature Angiogenesis

Cell movement of endothelial.

Endothelial cell development

Development of endothelial

Migration of endothelial cells

Migration of vascular

Movement of vascular

Formation of blood vessel

Growth of blood vessel

Neovascularization of organ

Neovascularization

Vascularization

Vascularization of absolute.

Tubulation of endothelial cells

Vascularization of eye
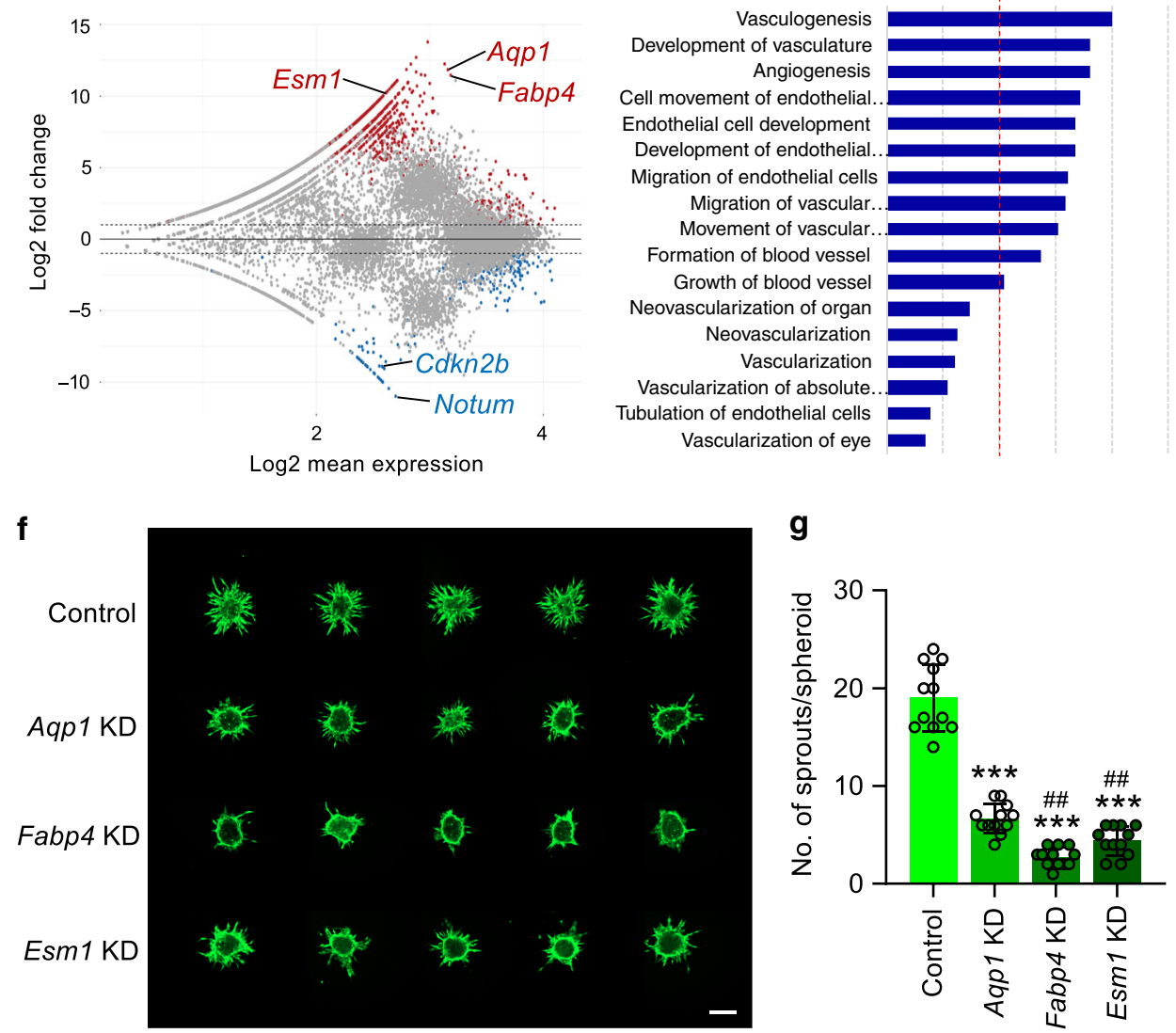

affects brain function also requires further investigation. While the debate on employing anti-VEGF therapy to manage cerebral edema in stroke and $\mathrm{HI}$ patients continues ${ }^{12,43-46}$, our results suggest that targeting this angiogenic pathway in HI patients may impede dura mater recovery and compromise its primary functions.

Therefore, our study provides an important molecular framework for dura mater vascular biology by identifying several key and organotypic EC molecules in dura mater BV maintenance and regeneration following injury, and establishes the functional role of these tissue-specific BVs in dura mater regeneration following $\mathrm{HI}$.

\section{Methods}

Mice. Specific pathogen-free (SPF) C57BL/6 J were purchased from DBL Co., Ltd. (Republic of Korea). Actin-GFP, Cx3cr1 DTR, and R26-tdTomato mice were purchased from Jackson Laboratory. VE-cadherin-Cre-ER ${ }^{\mathrm{T} 2} 21, V E G F-A^{+/ L a c Z} 47$ and VEGFR2 $2^{[\mathrm{fl} / f \mathrm{l} 22}, \mathrm{Tie}^{\mathrm{fl} / \mathrm{fl} 23}, \mathrm{Dll} 4^{\mathrm{fl} / \mathrm{fl} 24}$, and PDGFR $\beta$-Cre-ER ${ }^{\mathrm{T} 2} 48$ mice were transferred, established, and bred in SPF animal facilities at KAIST. All mice were maintained in the C57BL/6 background, housed under $12 \mathrm{hr}$ light $/ 12 \mathrm{hr}$ dark cycle, temperatures of $22 \pm 2{ }^{\circ} \mathrm{C}$ with $50 \pm 10 \%$ humidity, and fed with free access to a standard diet (PMI LabDiet) and water. In order to induce Cre activity in the Cre$\mathrm{ER}^{\mathrm{T} 2}$ mice, $2 \mathrm{mg}$ of tamoxifen (Sigma-Aldrich) dissolved in corn oil (SigmaAldrich) was injected intra-peritoneally (i.p.) at indicated time points for each experiment. Cre-ER ${ }^{\mathrm{T} 2}$ negative but flox/flox-positive mice among the littermates were defined as control mice for each experiment. Cre-mediated deletion 
Fig. 7 Dura mater ECs are superior to brain ECs in sprouting activity and show high expression of angiogenic molecules. $\mathbf{a}, \mathbf{b}$ Images and comparisons of the number of sprouts (length, $>50 \mu \mathrm{m}$ ) of EC spheroids derived from FACS-sorted primary ECs of dura mater (DM) and brain (B) at $12 \mathrm{hr}$ after incubation in collagen type I gel. Each dot indicates number of sprouts per spheroid. Each $n=6$ from two independent experiments. Vertical bars indicate mean \pm SD. $P=$ 0.0022 versus brain ECs by two-tailed Mann-Whitney $U$ test. Scale bars, $100 \mu \mathrm{m}$. $\mathbf{c}$ Highly magnified images of the left two panels in a showing robust sprouting (white arrowheads) and filopodia (yellow arrowheads) in EC spheroid from DM, whereas no sprouting and filopodia are seen in EC spheroid from B. Scale bars, $50 \mu \mathrm{m}$. d MA plot of significantly high-(1020 genes, red) and low (323 genes, blue)-expressing genes in dura mater ECs compared with brain ECs. Dotted horizontal lines demarcate twofold change between dura mater ECs and brain ECs. e IPA of 1343 differentially expressed genes in dura mater ECs compared with brain ECs. Ranked according to activation z score within Cardiovascular System Development and Function category. Vertical red-dotted line marks activation $z$ score threshold. $P<0.001$ for all pathways by right-tailed Fisher's Exact Test with Benjamini-Hochberg correction for multiple testing. f, $\mathbf{g}$ Representative images and comparisons of the number of sprouts (length, $>100 \mu \mathrm{m}$ ) from HUVEC spheroids at $24 \mathrm{~h}$ after incubation in collagen type I gel. siRNA-mediated KD was induced before spheroid formation. Scale bars, $200 \mu \mathrm{m}$. Each dot indicates number of sprouts per spheroid. Each $n=12$ from two independent experiments. Vertical bars indicate mean $\pm \mathrm{SD}$. ${ }^{\star \star \star} P<0.001$ versus control, ${ }^{\# \#} P<0.01$ versus Aqp1 KD by Kruskal-Wallis test.

efficiencies in both dura mater (84-88\%) and brain (81-85\%) were validated by histological analyses of the targeted proteins (Supplementary Fig. 15).

Mice were anesthetized with i.p. injection of a combination of anesthetics (80 $\mathrm{mg} / \mathrm{kg}$ ketamine and $12 \mathrm{mg} / \mathrm{kg}$ of xylazine) before any procedures or being sacrificed. Animal care and experimental procedures were performed under the approval from the Institutional Animal Care and Use Committee of KAIST (no. KA2018-42). All procedures and animal handlings were performed following the ethical guidelines for animal studies.

Photothrombotic injury. Mice at 8-10 weeks of age were anaesthetized with ketamine/xylazine immediately prior to surgery. Head fur was removed with pet hair clippers (Himax) and the incision site was sterilized with $70 \%$ ethanol. A surgical skin flap was created to expose the calvarium before the animal was mounted on a custom-built fast-scanning confocal microscope used for intravital imaging ${ }^{49}$. White light measured at $3 \mathrm{~mW}$ was aimed at the targeted injury site between the coronal and lambdoid sutures of the mouse calvarium and $100 \mu \mathrm{l}$ of Rose Bengal (15 mg/ml, Sigma-Aldrich) was injected into the retro-orbital vein. After 3 min of white light exposure to the targeted injury site, the surgical skin flap was sutured with a 5-0 (one metric) non-absorbable black braided silk suture (Ailee). Post-surgical care was performed under a heating lamp until mice showed complete recovery from anesthesia.

Histological analyses. After anesthesia, transcardial perfusion was performed into the mice with phosphate-buffered saline (PBS) followed by $4 \%$ paraformaldehyde (PFA, Merck). The dura mater-attached skull and brain of the PTI areas were harvested and the samples were then post-fixed at $4{ }^{\circ} \mathrm{C}$ in $4 \%$ PFA for $2 \mathrm{~h}$ for the dura mater and overnight for brain samples. Dura mater and pia mater samples were whole-mount stained. Brain samples were sectioned at $80 \mu \mathrm{m}$ thickness using a vibrating microtome (Leica Biosystems) prior to immunostaining. Samples were washed with PBS several times before blocking. After blocking with 5\% goat or donkey serum (Jackson ImmunoResearch) in $0.3 \%$ Triton-X 100 in PBS (PBST) for $1 \mathrm{~h}$, samples were incubated with the indicated primary antibodies diluted in the blocking solution at $4{ }^{\circ} \mathrm{C}$ overnight. After several washes with PBST, the samples were incubated at $4{ }^{\circ} \mathrm{C}$ overnight with the indicated fluorochrome-conjugated secondary antibodies diluted in the blocking buffer. The samples were washed with PBST and nuclei were stained with 4',6-diamidino-2-phenylindole (DAPI) (Invitrogen). After washing with PBS, samples were mounted with Vecta-shield (Vector Laboratories). Images were obtained using Zeiss LSM 800 or LSM 880 confocal microscope (Carl Zeiss). The following primary antibodies were used in the immunostaining of mouse samples: anti-CD31 (hamster monoclonal, MAB1398Z, Merck), anti-pVEGFR2 (Tyr1175, rabbit monoclonal, 2478, Cell Signaling Technology), anti-pERK (Thr202/Tyr204, rabbit monoclonal, 4370, Cell Signaling Technology), anti-pAkt (Ser473, rabbit monoclonal, 4060, Cell Signaling Technology), anti-VE-PTP (rabbit polyclonal, generously provided by Dietmar Vestweber), anti-VEGFR2 (goat polyclonal, AF644, R\&D), anti-VEGFR3 (goat polyclonal, AF743, R\&D), anti-Tie2 (goat polyclonal, AF762, R\&D), anti-Dll4 (goat polyclonal, AF1389, R\&D), anti-Ang2 ${ }^{50}$, anti-NG2 (rabbit polyclonal, AB5320, Merck), anti-F4/80 (rat monoclonal, MCA497, Bio-Rad), anti-LYVE-1 (rabbit polyclonal, 11-034, Angiobio), anti-VEGF ${ }_{164}$ (goat polyclonal, AF-493-NA, R\&D), anti-Osterix (rabbit polyclonal, ab22552, Abcam), anti-collagen type IV (rabbit polyclonal, ab6586, Abcam), anti-vimentin (chicken polyclonal, AB5733, Merck) and anti-neurofilament heavy polypeptide (rabbit polyclonal, ab8135, Abcam). The following primary antibodies were used in the immunostaining of human samples: anti-VEGFR2 (goat polyclonal, AF357, R\&D) and anti-CD31 (rabbit polyclonal, ab28364, Abcam). Alexa Fluor 488-, Alexa Fluor 594-, Alexa Fluor 647-conjugated secondary antibodies were purchased from Jackson ImmunoResearch. All the antibodies used in our study were validated for the species and applications. $\beta$-gal staining of $V E G F-A^{+/ L a c Z}$ samples was performed as previously described ${ }^{51}$. In brief, samples were washed and permeabilized with staining buffer $(2 \mathrm{mM}$ $\mathrm{MgCl}_{2}, 0.01 \%$ sodium deoxycholate, and $0.02 \% \mathrm{NP}-40$ in PBS) and stained for $4 \mathrm{~h}$ in $1 \mathrm{mg} / \mathrm{ml} \mathrm{X}$-gal in staining buffer supplemented with $5 \mathrm{mM}$ potassium ferricyanide and $5 \mathrm{mM}$ potassium ferrocyanide. Samples were washed with PBS several times before immunostaining.

Morphometric analysis. Morphometric measurements were performed using ImageJ software (NIH) or ZEN 2012 software (Carl Zeiss). To determine vascular density and distribution of collagen IV in the mice with PTI or sham operation, MFI of CD31+ or collagen IV + was measured in dura mater $\left(2.5 \mathrm{~mm}^{2}\right)$, pia mater $\left(4.0 \mathrm{~mm}^{2}\right)$, and brain $\left(0.36 \mathrm{~mm}^{2}\right)$ of the injury or putative injury areas. To determine vascular density and distribution of collagen IV in the genetically modified mice, MFI of CD31+ or collagen IV was measured in dura mater $\left(3.6 \mathrm{~mm}^{2}\right)$, pia mater $\left(5.0 \mathrm{~mm}^{2}\right)$, and brain $\left(1.0 \mathrm{~mm}^{2}\right)$. To measure density of LYVE1+ or F4/80+ macrophages in the dura mater of genetically modified mice, each MFI was measured in area $\left(0.36 \mathrm{~mm}^{2}\right)$ of three regions per sample. To measure density of LYVE1+ or F4/80+ macrophages in the mice with PTI or sham operation, each MFI was measured in injured area of dura mater $\left(2.5 \mathrm{~mm}^{2}\right)$, pia mater $\left(4.0 \mathrm{~mm}^{2}\right)$, and brain $\left(0.36 \mathrm{~mm}^{2}\right)$. To measure VE-PTP density in VEGFR2+ BVs, MFI of VEPTP was measured in the dura mater $\left(0.36 \mathrm{~mm}^{2}\right)$ and brain $\left(0.36 \mathrm{~mm}^{2}\right)$. To measure density of perivascular cells in the dura mater, MFI of NG2+ cells was measured in the injury area $\left(0.36 \mathrm{~mm}^{2}\right)$. To determine density of fibroblasts, MFI of vimentin + cells was measured in dura mater $\left(1.2 \mathrm{~mm}^{2}\right)$ and ear skin $(0.20$ $\mathrm{mm}^{2}$ ). To determine vascular density in the ear skin, MFI of CD31 was measured in circumferential region $\left(0.10 \mathrm{~mm}^{2}\right)$ of punch hole injury. To determine EB leakage, IFI of EB was measured in injured area of dura mater $\left(3.6 \mathrm{~mm}^{2}\right)$, pia mater $\left(4.0 \mathrm{~mm}^{2}\right)$, and brain $\left(1.0 \mathrm{~mm}^{2}\right)$. To measure VEGFR2 in the BVs of human dura mater and brain, each MFI of VEGFR2 + or CD31+ was measured in the indicated area of dura mater $\left(0.36 \mathrm{~mm}^{2}\right)$ and brain $\left(0.36 \mathrm{~mm}^{2}\right)$.

EdU incorporation assay for proliferating ECs. To detect proliferating ECs, $5 \mathrm{mg}$ of 5-ethynyl-2'-deoxyuridine (EdU, A10044, Invitrogen) was dissolved in $1 \mathrm{ml}$ of Milli-Q water as a stock solution. Then, $200 \mu \mathrm{l}$ of the stock solution per mice was injected i.p. every day, starting from 1 day prior to PTI and for 4 days before analysis. Tissues were isolated and processed as described above. EdU-incorporated cells were detected with the Click-iT EdU Alexa Fluor 488 Assay Kit (Invitrogen) according to the manufacturer's protocol.

Lectin perfusion assay. Mice were anaesthetized immediately prior to retroorbital injection of fluorescein isothiocyanate (FITC)-conjugated lectin (SigmaAldrich, $100 \mu \mathrm{g} / 100 \mu \mathrm{l})$. Animals were killed $30 \mathrm{~min}$ after injection, processed and immunostained as described above. FITC was detected in the $488 \mathrm{~nm}$ channel but pseudo-colored to red using ZEN software (Carl Zeiss). Percentage of perfused BV was calculated as the lectin $+/ C D 31+$ perfused $B V$ area divided by total CD $31+B V$ areas in the putative injury area $\left(1.44 \mathrm{~mm}^{2}\right)$ of dura mater.

Evans Blue leakage assay. Mice were anaesthetized immediately prior to retroorbital injection of Evans Blue solution (Merck, 1\% v/w, $200 \mu \mathrm{l}$ ). Animals were killed $1 \mathrm{~h}$ after injection as described above. In order to distinguish vascular leakage in the dura mater from the calvarial bone marrow, dura mater tissues were separated from the calvarial bone after overnight decalcification with $10 \%$ ethylenediaminetetraacetic acid prior to immunostaining.

Treatment of blocking antibody. For VEGFR2 and PDGFR $\beta$ blockade, we used VEGFR2- and PDGFR $\beta$-neutralizing antibodies DC101 and APB5 ${ }^{28}$, respectively. A hybridoma cell line that produces DC101 was purchased from American Type Culture Collection (ATCC). A hybridoma cell line that produces APB5 was generously provided by Akiyoshi Uemura (Nagoya City University, Japan). Hybridoma cells were grown in serum-free medium. Recombinant proteins in the supernatants were purified by column chromatography with Protein A agarose gel (Oncogene). After purification, the recombinant proteins were quantified using the Bradford assay and confirmed by Coomassie blue staining after sodium dodecyl sulfate-polyacrylamide gel electrophoresis. DC101 (40 mg/kg) or APB5 $(25 \mathrm{mg} / \mathrm{kg})$ 
a

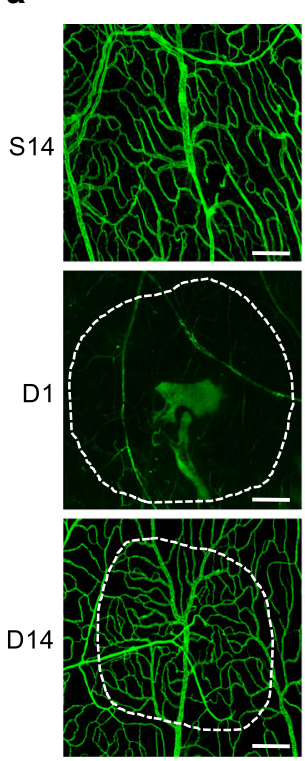

CD31
Dura mater

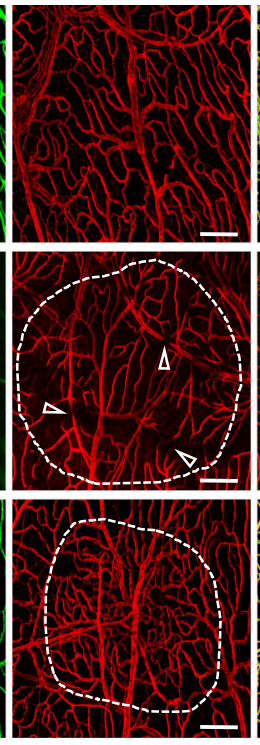

Collagen IV
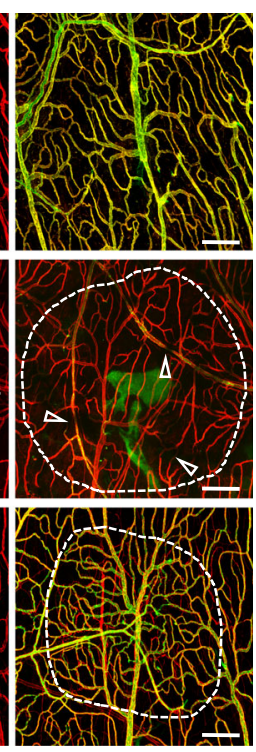

Collagen IV CD31

C
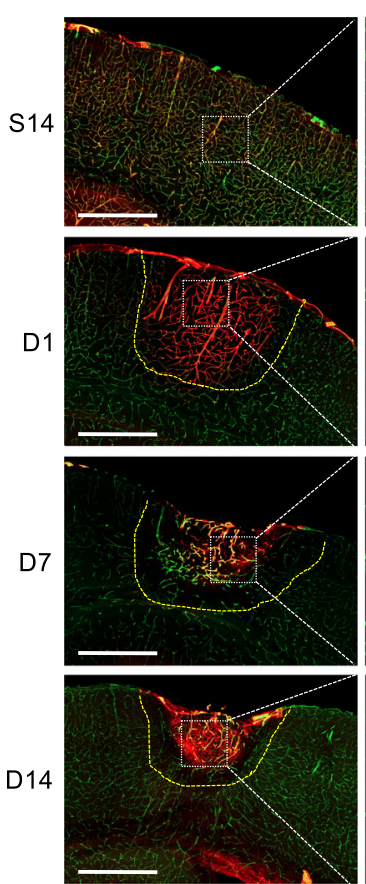

Collagen IV CD31

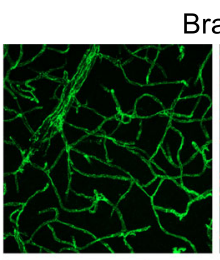

Brain
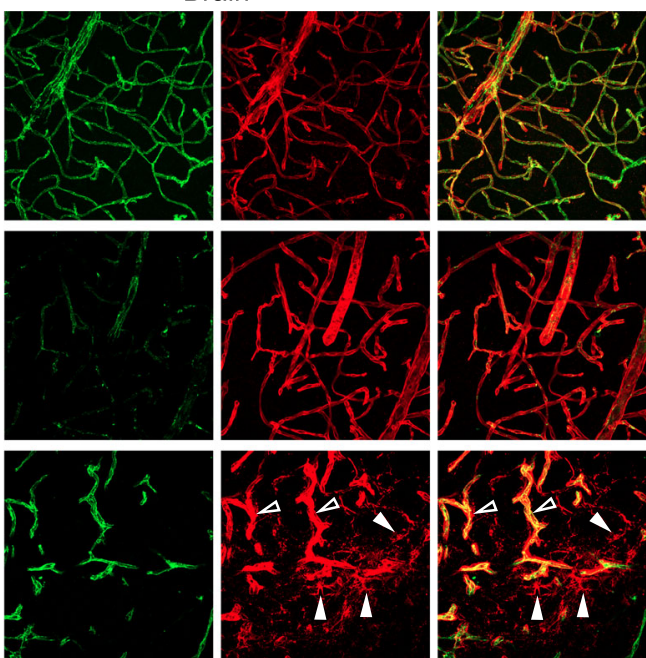

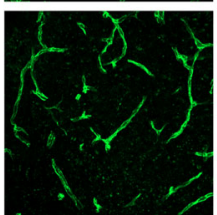

CD31

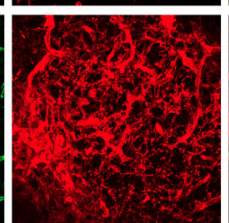

Collagen IV b
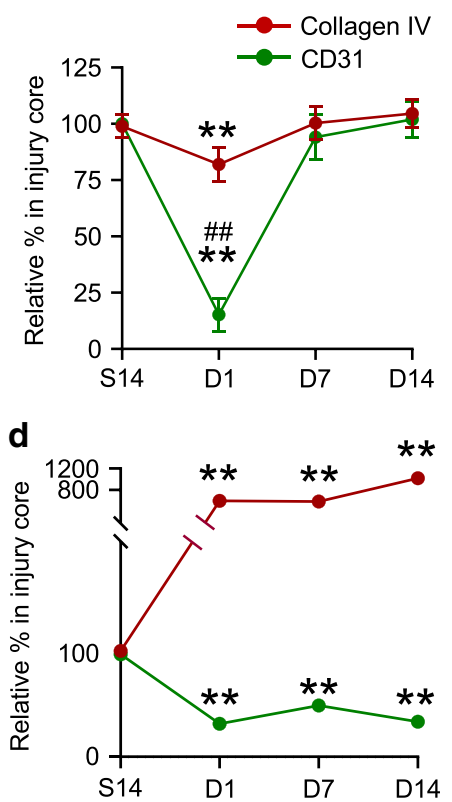

Fig. 8 Rapid recovery of BV basement membrane in the injured dura mater but not in the injured brain after PTI. a, b Representative images and comparisons of distributions of collagen IV along CD31+BVs in putative injury area (white dotted-lined circle) at indicated days in the dura mater of adult mice after PTI. Empty arrowheads indicate broken alignment of collagen IV. Scale bars, $200 \mu \mathrm{m} . n=4$ mice/group from two independent experiments. Mean fluorescence intensity (MFI) of collagen IV per MFI of CD31 in the putative injury area at S14 is regarded as 100\%. Dots and error bars indicate mean

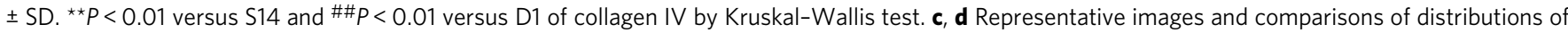
collagen IV and CD31+BVs in putative injury area (white dotted line) at indicated days in the brain of adult mice after PTI. Scale bars, $500 \mu \mathrm{m}$. Each indicated box area is magnified and arrayed in the right panels. Empty arrowheads indicate increased collagen IV in perivascular areas, whereas white arrowheads indicate collagen IV accumulation in avascular areas. $n=4$ mice/group from two independent experiments. MFI of collagen IV or MFI of CD31 in the putative injury area at S14 is regarded as 100\%. Dots and error bars indicate mean \pm SD. ${ }^{\star \star} P<0.01$ versus S14 by Kruskal-Wallis test.

and an equal amount of the control antibody (IgG-Fc) was i.p. injected into the indicated mice every other day until mice were sacrificed for analyses.

Human dura mater and brain samples. Specimens of dura mater and brain cortex obtained from four deceased individuals who suffered fatal HI from vertical falls were autopsied with court-issued warrants at the request of the public prosecutor The Institutional Review Board of Chonnam National University Medical School and Hospital (Gwangju, Republic of Korea) approved all autopsies as research activity involving non-living, post-mortem subjects in accordance with ethical regulations and waived the requirement to obtain informed consent owing to the inaccessibility to and unavailability of personal identification information and 
a

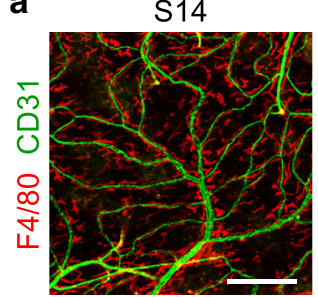

C

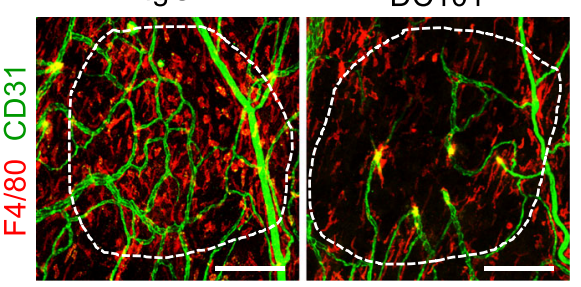

b

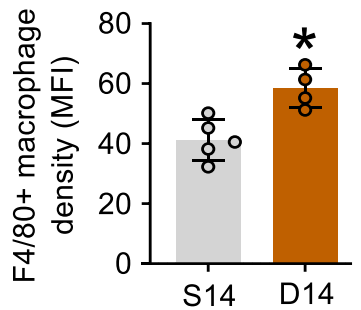

d

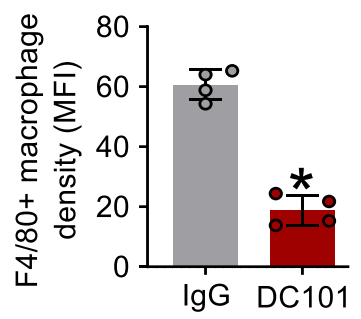

e

CD31

Vimentin

Merged
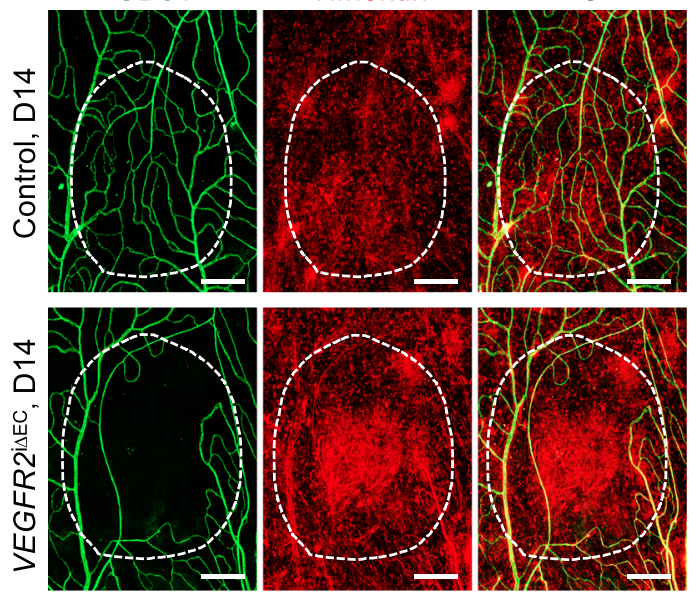

f

Control

VEGFR2 $2^{\mathrm{i} \triangle \mathrm{EC}}$

g

CD31

h
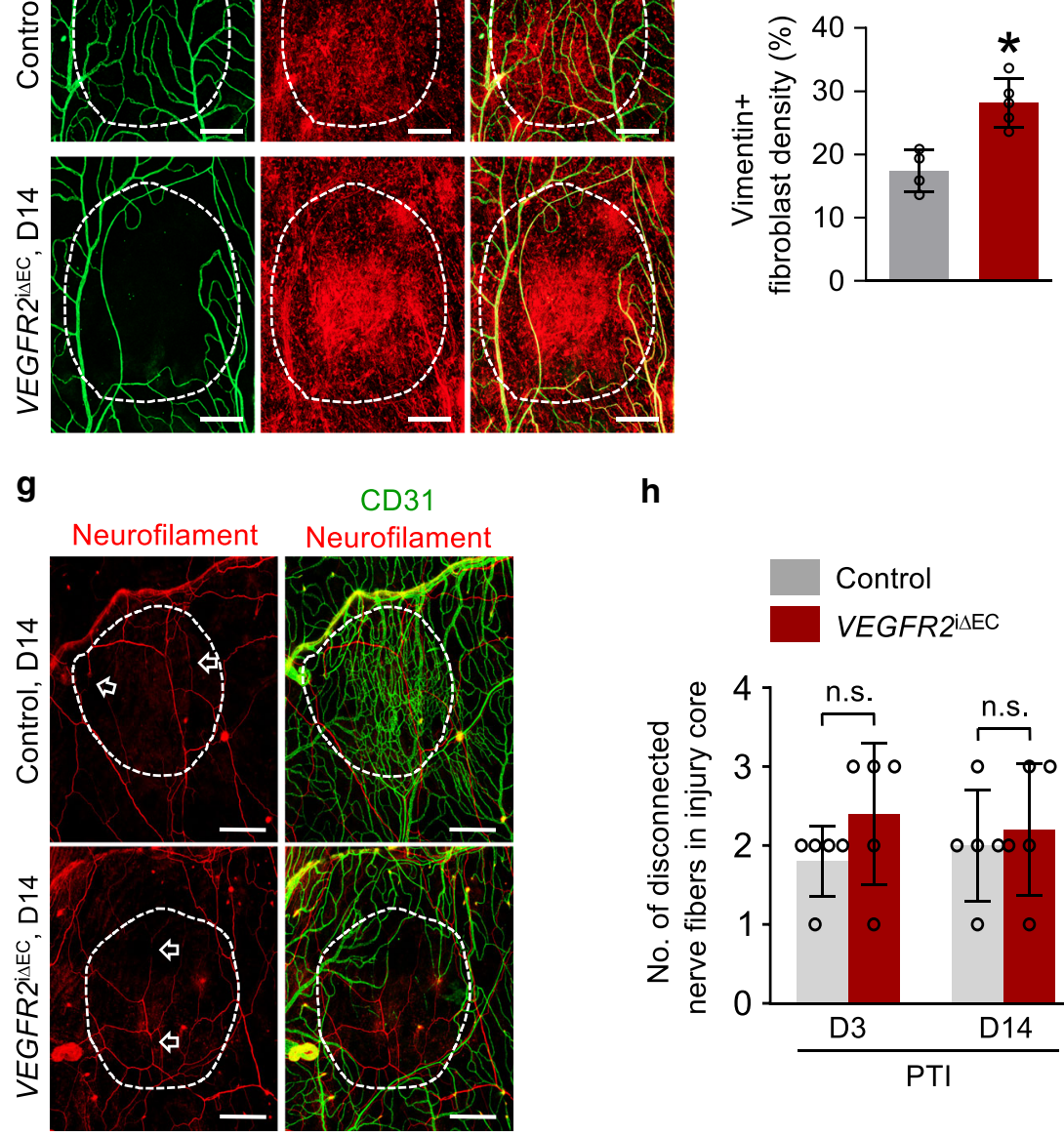

testing for heritable traits of the deceased (no. 2019-001). Accordingly, all specimens and data, with the exception of age, gender, and medical histories, were anonymized prior to all analyses. Dura mater samples were whole-mount immunostained, whereas brain cortex samples were sectioned at $100 \mu \mathrm{m}$ with a vibrating microtome (Leica Biosystems) prior to immunostaining.

qRT-PCR analyses of whole dura mater tissue. Dura mater was isolated as mentioned above without any transcardial perfusion. Dura mater was removed from the attaching calvarial bone by submerging the tissue in ice-cold PBS and

peeling the organ with fine forceps. Tissue was mechanically dissociated using a Precellys 24 tissue homogenizer (Bertin Technologies) until cells were completely lysed. RNA was extracted using RNeasy Micro kit (Qiagen) or Trizol RNA

extraction kit (Invitrogen). A total of $1 \mu \mathrm{g}$ of extracted RNA was transcribed into cDNA using GoScript Reverse Transcription Kit (Promega). Quantitative real-time PCR was performed using FastStart SYBR Green Master mix (Roche) and S1000 Thermocycler (Bio-Rad) with the indicated primers. The primers were designed using Primer-BLAST or adopted from previously published studies: Vegfa $\left(5^{\prime}-\right.$ TGCCAAGTGGTCCCAGGCTGC-3'; 5'-CCTGCACAGCGCATCAGCGG-3'); Vegfc (5'-GAGGTCAAGGCTTTTGAAGGC-3'; 5'- 
Fig. 9 Vascular regeneration is required for recovery of perivascular macrophages and normalization of fibroblasts in dura mater after PTI. a, b Representative images and comparisons of the distribution of $\mathrm{F} 4 / 80+$ macrophages in the putative injury area (white dotted-lined circle) of dura mater at S14 and D14 in adult mice. Scale bars, $200 \mu \mathrm{m}$. Each dot indicates a mean value obtained from one mouse and $n=5$ (S14), $n=4$ (D14) mice/group from two independent experiments. Vertical bars indicate mean \pm SD. ${ }^{\star} P=0.0159$ versus S14 by two-tailed Mann-Whitney U test. MFI, mean fluorescence intensity. c, $\mathbf{d}$ Representative images and comparisons of the distribution of F4/80+ macrophages in the putative injury area (white dotted-lined circle) of dura mater at D14 in adult mice that were treated with IgG-Fc or DC101 (daily i.p. injections of $40 \mathrm{mg} / \mathrm{kg}$ of body weight for 6 days). Note substantial inhibition of F4/80+ macrophage distribution in the injury area with DC101 treatment. Scale bars, $200 \mu \mathrm{m}$. Each dot indicates a mean value obtained from one mouse and $n=4$ mice/group from two independent experiments. Vertical bars indicate mean \pm SD. ${ }^{\star} P=0.0286$ versus lgG-Fc by two-tailed Mann-Whitney $U$ test. MFI, mean fluorescence intensity. e, $\mathbf{f}$ Representative images and comparisons of the distribution of vimentin+ fibroblasts in the putative injury area (white dotted-lined circle) of dura mater in control and VEGFR2 ${ }^{2} E C$ mice at D14. Scale bars, $200 \mu \mathrm{m}$. Each dot indicates a mean value obtained from one mouse and $n=4$ (control), $n=5\left(V E G F R 2^{i \triangle E C}\right)$ mice/group from two independent experiments. Vertical bars indicate mean \pm SD. ${ }^{\star} P=$ 0.0159 versus control by two-tailed Mann-Whitney $U$ test. $\mathbf{g}$, $\mathbf{h}$ Representative images and comparisons of the number of broken neurofilament+nerve fibers (arrows) in the putative injury area (white dotted-lined circle) of dura mater in control and VEGFR2i $\triangle E C$ mice at D3 and D14. Scale bars, $200 \mu$ m. Each dot indicates a mean value obtained from one mouse and $n=5$ mice/group from three independent experiments. Vertical bars indicate mean \pm SD. n.s. not significant by two-tailed Mann-Whitney $U$ test.

CTGTCCTGGTATTGAgGGTGG-3'); Pgf (5'-TCTGCTGGGAACAACTCAAC A-3'; $5^{\prime}$-GTGAGACACCTCATCAGGGTAT-3'). Gapdh (5'-AGGTCGGTGTGAACGGATTTG-3'; $5^{\prime}$-TGTAGACCATGTAGTTGAGGTCA-3') was used as a reference gene and the results were presented as relative expressions to control. Primer reaction specificity was confirmed by melting curve analysis. Relative gene expression was analyzed by $\Delta \Delta \mathrm{Ct}$ method using the CFX Manager software (BioRad).

ELISA for quantification of VEGF-A. Dura mater was separated from the calvarial bone as described above. Brain cortex was separated from the rest of the brain using a surgical scalpel. Tissues were mechanically dissociated as described above and protein was extracted in Cell Extraction Buffer (ThermoFischer Scientific). Sample protein concentrations were measured and normalized with a Pierce BCA Protein Assay Kit (ThermoFisher Scientific) and the concentration of VEGF-A in tissue extracts was measured using a Mouse VEGF-A Quantikine ELISA Kit (MV00, R\&D).

\section{Depletion of CX3CR1 ${ }^{+}$monocytes/macrophages. To deplete CX3CR1 ${ }^{+}$} monocytes and macrophages in CX3CR1-DTR mice receiving PTI, $1 \mu \mathrm{g}$ of diphtheria toxin (Merck) diluted in PBS was injected every other day, beginning from 1 day before PTI until mice were sacrificed at 7 days after PTI for analysis. Control mice received injections of PBS.

\section{Spheroid-based sprouting assay. EC spheroids were generated by incubating} $\sim 1000$ FACS-sorted primary dura mater and brain ECs in endothelial growth media (EGM2, Lonza) containing $0.25 \%$ methylcellulose as hanging-drops overnight ${ }^{35}$. The spheroids were then collected and embedded in $2 \mathrm{mg} / \mathrm{ml}$ collagen type I (Corning) with mouse VEGF-A-supplied (50 ng/ml) EGM for $24 \mathrm{~h}$. At the end of the incubation, spheroid was stained with cell tracker (Molecular Probes, $1.5 \mu \mathrm{M}$, $37^{\circ} \mathrm{C}, 30 \mathrm{~min}$ ). The spheroids were then fixed in $4 \%$ PFA for $15 \mathrm{~min}$ at RT and imaged using a Zeiss LSM 800 confocal microscope (Carl Zeiss).

siRNA-mediated knockdown. Human umbilical vein ECs (HUVECs) were maintained in EGM2 media (Lonza). For small RNA interference (siRNA), oligonucleotides against Aqp1 (358-2, Bioneer), against Fabp4 (2167-1, Bioneer), against Esm1 (11082-2, Bioneer), or scrambled siRNA for negative control were used. HUVECs with a 40-60\% confluence were transfected with siRNAs using Lipofectamine RNAiMAX (Invitrogen) in OptiMEM media. Culture media was changed 4-6 hr after transfection.

Magnetic-associated and fluorescence-activated cell sorting. To sort dura mater or brain ECs, $\mathrm{CD} 45^{+}$hematopoietic or $\mathrm{CD} 45^{-}$non-hematopoietic cells by FACS, tissues were isolated as described above and dissociated in collagenase type I (1 mg/ml, Worthington), dispase $(1 \mathrm{mg} / \mathrm{ml}$, Gibco), and DNase I $(0.1 \mathrm{mg} / \mathrm{ml}$, Merck) dissolved in DMEM/F12 supplemented with 5\% fetal bovine serum (FBS) at $37^{\circ} \mathrm{C}$ for $30 \mathrm{~min}$. Cell suspensions went on RBC lysis by suspension in ACK lysis buffer (Gibco) for $5 \mathrm{~min}$ on ice and blocked with mouse anti-CD16/CD32 (553141, $\mathrm{BD}$ Bioscience) before being incubated for $20 \mathrm{~min}$ with indicated antibodies in FACS buffer (2\% FBS in PBS). After several washes, brain ECs were enriched using automatic magnetic-associated cell sorting (MACS, Miltenyi-Biotec) prior to be being stained with fluorochrome-conjugated primary antibodies. Cell sorting was performed with FACS Aria II (Beckton Dickinson). Dead cells were excluded using DAPI (Sigma-Aldrich) staining and cell doublets were systematically excluded. The following antibodies were used for MACS: biotin anti-mouse CD31 (rat monoclonal, 130-119-562, Miltenyi-Biotec); anti-biotin microbeads (130-090-485). The following antibodies were used for FACS: PE anti-mouse CD31 (rat monoclonal,
102508, Biolegend); APC anti-mouse CD45 (rat monoclonal, 103112, Biolegend); FITC anti-mouse TER-119 (rat monoclonal, 116206, Biolegend).

Bulk RNA-sequencing. Bulk RNA-sequencing of sorted ECs was performed by obtaining the alignment file. In brief, reads were mapped using TopHat software tool. The alignment file was used to assemble transcripts, estimate their abundances, and detect differential expression of genes or isoforms using cufflinks. The Ingenuity Pathway Analysis tool (QIAGEN) was used to further evaluate the data in the context of biological categories and canonical signaling. The significance of biological categories and canonical signaling was tested by the

Benjamini-Hochberg procedure, which adjusts the $P$ value to correct for multiple comparisons, and their activation or inhibition was determined with reference to activation $z$ scores.

Parabiosis model. Pairs of 8-10-week-old wild-type and actin-GFP ${ }^{+}$mice were subjected to parabiotic surgery. Mice were anesthetized by intramuscular injection of the combination of anesthetics, and then surgically joined from shoulder to femur. At the indicated weeks after parabiotic surgery, $\mathrm{GFP}^{+}$cells in the injured tissues of wild-type mice were examined.

Single-cell-RNA sequencing using droplet-based method. Single-cell suspensions were prepared as described above, diluted to $1000 \mathrm{cells} / \mu \mathrm{l}$ in PBS, and processed with the $10 \times$ Chromium platform using $10 \times$ Chromium single-cell $3^{\prime}$ reagents, following manufacturer's protocols. In brief, required number of cells were loaded to $10 \times$ chips with RT reagent mix and RT primers. After partitioning cells into Gel Beads in Emulsion, transcripts from single cells were barcoded and reverse-transcribed to single-cell cDNA libraries. Then, cDNA libraries were enzymatically fragmented, end-repaired and A-tailed. After double-sized size selection using SPRI beads (Beckman Coulter), adapters were ligated and sampleindex PCR was performed. Products went through another round of double-sized size selection using SPRI beads. For quality check of final libraries, they were diluted to 10 -fold and run on Agilent Bioanalyzer High Sensitivity Chip. Single-cell libraries that passed quality control were then sequenced on Illumina HiSeq-X platform.

Construction of raw expression matrix. Raw base call (BCL) files from Illumina sequencers were first demultiplexed into FASTQ files using mkfastq function in Cell Ranger 3.1.0 toolkit from 10X Genomics. Then, reads were mapped to $\mathrm{mm} 10$ reference genome (v3.0.0, 10X Genomics). Next, using R package 'Seurat' (v3.1.0) ${ }^{52}$, raw expression matrices were built from aligned reads by Read10X function in Seurat. To remove potential doublets formed by cells with distinct transcriptome, Scrublet (Single-Cell Remover of Doublets) ${ }^{53}$ was used. Scrublet generates simulated doublets from randomly selected cells in the data set and constructs nearest neighbor classifier to identify potential doublets. In addition, cells that have high percentage of mitochondrial unique molecular identifier (UMI) counts $(>7 \%)$, and cells that have too low or too high number of detected genes $(>1000$ and $>6000)$ were discarded from the data sets. Only cells that passed such stringent quality control processes were used for further analysis.

Integrating data sets and clustering analysis. From the four data sets, 2500 cells that all passed quality controls, were random sampled, yielding 10,000 cells in total. To observe whether batch effects exist, random sampled cells were first merged without any imputation-based methods. After initial clustering, clusters were not separated by batches, suggesting minimal effect arising from experimental batches. Then, the expression matrix of merged data set was first normalized by the NormalizeData function in Seurat, which normalizes the read counts by dividing UMI counts for each gene in a given cell by the sum of all UMI counts in that cell, 
multiplies 10,000 and transforms into log scales. Genes that are highly variable in the data set were identified by FindVariableFeatures function in Seurat with options (selection.method = "vst", nfeatures $=2500$ ). Data were scaled and variables such as mitochondrial percentages and total number of UMI counts were regressed by ScaleData function in Seurat. Dimensionality of the data was reduced by performing principal component analysis with the highly variable genes identified on the scaled data. Top 30 principal components were used for uniform manifold approximation and projection to visualize data into two-dimensional space. For the identification of clusters, FindNeighbors and FindClusters functions in Seurat were used with 30 principal components and with resolution parameter of 0.6 , respectively.

Identifying differentially expressed genes. Cluster-specific DEG were identified using the FindMarkers function in Seurat. The test MAST (Model-based Analysis of Single-cell Transcriptomics) ${ }^{54}$ was used for differential expression testing, which utilizes the hurdle model fit for single-cell data. Top 100 DEGs for each cluster in the integrated data set were used as input in Immgen (http://www.immgen.org) data browser to characterize and annotate cell populations. Finally, to visualize DEGs identified between clusters, DoHeatmap function for heatmaps and VlnPlot function for violin plots in Seurat were used with default parameters.

Statistical analysis. No statistical methods were used to predetermine sample size. The experiments were randomized and investigators were blinded to allocation during experiments and outcome analyses. All values are presented as mean \pm standard deviation (SD). Statistical significance was determined by the two-sided Mann-Whitney $U$ test between two groups or the two-way analysis of variance by Kruskal-Wallis multiple comparisons test for multiple-group comparison. Statistical analyses were performed using GraphPad Prism 8.0 (GraphPad Software). Statistical significance was set at $P<0.05$.

Reporting summary. Further information on research design is available in the Nature Research Reporting Summary linked to this article.

\section{Data availability}

RNA-sequencing data are available in the National Center for Biotechnology Information's Gene Expression Omnibus under accession numbers GSE145973 (scRNAseq) [https://www.ncbi.nlm.nih.gov/geo/query/acc.cgi?acc=GSE145973] and GSE138561 (bulk RNAseq) [https://www.ncbi.nlm.nih.gov/geo/query/acc.cgi? acc=GSE138561]. All other data that support the findings of this study are available from the corresponding author upon reasonable request. Source data are provided with this paper.

Received: 10 December 2019; Accepted: 6 July 2020; Published online: 31 July 2020

\section{References}

1. Roozenbeek, B., Maas, A. I. R. \& Menon, D. K. Changing patterns in the epidemiology of traumatic brain injury. Nat. Rev. Neurol. 9, 231-236 (2013).

2. Nguyen, R. et al. The international incidence of traumatic brain injury: a systematic review and meta-analysis. Can. J. Neurol. Sci. 43, 774-785 (2016).

3. Blennow, K. et al. Traumatic brain injuries. Nat. Rev. Dis. Prim. 2, 16084 (2016).

4. Rusnak, M. Traumatic brain injury: giving voice to a silent epidemic. Nat. Rev. Neurol. 9, 186-187 (2013).

5. Dekosky, S. T., Blennow, K., Ikonomovic, M. D. \& Gandy, S. Acute and chronic traumatic encephalopathies: pathogenesis and biomarkers. Nat. Rev. Neurol. 9, 192-200 (2013).

6. Decimo, I., Fumagalli, G., Berton, V., Krampera, M. \& Bifari, F. Meninges: from protective membrane to stem cell niche. Am. J. Stem Cells 1, 92-105 (2012).

7. Weller, R. O., Sharp, M. M., Christodoulides, M., Carare, R. O. \& Møllgård, K. The meninges as barriers and facilitators for the movement of fluid, cells and pathogens related to the rodent and human CNS. Acta Neuropathol. 135 363-385 (2018).

8. Mecheri, B., Paris, F. \& Lübbert, H. Histological investigations on the dura mater vascular system of mice. Acta Histochem. 120, 846-857 (2018).

9. Mastorakos, P. \& McGavern, D. The anatomy and immunology of vasculature in the central nervous system. Sci. Immunol. 4, eaav0492 (2019).

10. Rua, R. \& McGavern, D. B. Advances in meningeal immunity. Trends Mol. Med. 24, 542-559 (2018)

11. Zhang, Y., Xiong, Y., Mahmood, A., Zhang, Z. G. \& Chopp, M. Angiogenesis and functional recovery after traumatic brain injury. in Vascular Mechanisms in CNS Trauma 141-156 (Springer New York, 2014).
12. Salehi, A., Zhang, J. H. \& Obenaus, A. Response of the cerebral vasculature following traumatic brain injury. J. Cereb. Blood Flow. Metab. 37, 2320-2339 (2017).

13. Xiong, Y., Mahmood, A. \& Chopp, M. Angiogenesis, neurogenesis and brain recovery of function following injury. Curr. Opin. Investig. Drugs 11, 298-308 (2010).

14. Russo, M. V., Latour, L. L. \& McGavern, D. B. Distinct myeloid cell subsets promote meningeal remodeling and vascular repair after mild traumatic brain injury. Nat. Immunol. 19, 442-452 (2018).

15. Labat-gest, V. \& Tomasi, S. Photothrombotic ischemia: a minimally invasive and reproducible photochemical cortical lesion model for mouse stroke studies. J. Vis. Exp. 50370 (2013).

16. Jha, R. M., Kochanek, P. M. \& Simard, J. M. Pathophysiology and treatment of cerebral edema in traumatic brain injury. Neuropharmacology 145, 230-246 (2019).

17. Simons, M., Gordon, E. \& Claesson-Welsh, L. Mechanisms and regulation of endothelial VEGF receptor signalling. Nat. Rev. Mol. Cell Biol. 17, 611-625 (2016).

18. Saharinen, P., Eklund, L. \& Alitalo, K. Therapeutic targeting of the angiopoietin-TIE pathway. Nat. Rev. Drug Discov. 16, 635-661 (2017).

19. Benedito, R. et al. The notch ligands Dll4 and Jagged1 have opposing effects on angiogenesis. Cell 137, 1124-1135 (2009).

20. Bray, S. J. Notch signalling in context. Nat. Rev. Mol. Cell Biol. 17, 722-735 (2016).

21. Okabe, K. et al. Neurons limit angiogenesis by titrating VEGF in retina. Cell 159, 584-596 (2014).

22. Sison, K. et al. Glomerular structure and function require paracrine, not autocrine, VEGF-VEGFR-2 signaling. J. Am. Soc. Nephrol. 21, 1691-1701 (2010).

23. Savant, S. et al. The orphan receptor tiel controls angiogenesis and vascular remodeling by differentially regulating tie2 in tip and stalk cells. Cell Rep. 12, 1761-1773 (2015).

24. Hozumi, K. et al. Delta-like 4 is indispensable in thymic environment specific for T cell development. J. Exp. Med. 205, 2507-2513 (2008).

25. Bäumer, S. et al. Vascular endothelial cell-specific phosphotyrosine phophatase (VE-PTP) activity is required for blood vessel development. Blood 107, 4754-4762 (2006).

26. Hayashi, M. et al. VE-PTP regulates VEGFR2 activity in stalk cells to establish endothelial cell polarity and lumen formation. Nat. Commun. 4, 1672 (2013).

27. Armulik, A., Genové, G. \& Betsholtz, C. Pericytes: developmental, physiological, and pathological perspectives, problems, and promises. Dev. Cell 21, 193-215 (2011)

28. Uemura, A. et al. Recombinant angiopoietin-1 restores higher-order architecture of growing blood vessels in mice in the absence of mural cells. J. Clin. Invest. 110, 1619-1628 (2002).

29. Schain, A. J. et al. Activation of pial and dural macrophages and dendritic cells by cortical spreading depression. Ann. Neurol. 83, 508-521 (2018).

30. Cho, C.-H. et al. Angiogenic role of LYVE-1-positive macrophages in adipose tissue. Circ. Res. 100, e47-e57 (2007).

31. Dick, S. A. et al. Self-renewing resident cardiac macrophages limit adverse remodeling following myocardial infarction. Nat. Immunol. 20, 29-39 (2019).

32. Chakarov, S. et al. Two distinct interstitial macrophage populations coexist across tissues in specific subtissular niches. Science 15, 363 (2019).

33. Corliss, B. A., Azimi, M. S., Munson, J. M., Peirce, S. M. \& Murfee, W. L. Macrophages: an inflammatory link between angiogenesis and lymphangiogenesis. Microcirculation 23, 95-121 (2016).

34. Wynn, T. A. \& Vannella, K. M. Macrophages in tissue repair, regeneration, and fibrosis. Immunity 44, 450-462 (2016).

35. Choi, D. et al. Laminar flow downregulates Notch activity to promote lymphatic sprouting. J. Clin. Invest. 127, 1225-1240 (2017).

36. Inai, $T$. et al. Inhibition of vascular endothelial growth factor (VEGF) signaling in cancer causes loss of endothelial fenestrations, regression of tumor vessels, and appearance of basement membrane ghosts. Am. J. Pathol. 165, 35-52 (2004).

37. Xiong, Y., Mahmood, A. \& Chopp, M. Animal models of traumatic brain injury. Nat. Rev. Neurosci. 14, 128-142 (2013).

38. Namjoshi, D. R. et al. Towards clinical management of traumatic brain injury: a review of models and mechanisms from a biomechanical perspective. Dis. Model. Mech. 6, 1325-1338 (2013).

39. Ma, X. et al. Animal models of traumatic brain injury and assessment of injury severity. Mol. Neurobiol. 56, 5332-5345 (2019).

40. Louveau, A. et al. Structural and functional features of central nervous system lymphatic vessels. Nature 523, 337-341 (2015).

41. Aspelund, A. et al. A dural lymphatic vascular system that drains brain interstitial fluid and macromolecules. J. Exp. Med. 212, 991-999 (2015).

42. Ahn, J. H. et al. Meningeal lymphatic vessels at the skull base drain cerebrospinal fluid. Nature 572, 62-66 (2019). 
43. Thau-Zuchman, O., Shohami, E., Alexandrovich, A. G. \& Leker, R. R. Vascular endothelial growth factor increases neurogenesis after traumatic brain injury. J. Cereb. Blood Flow. Metab. 30, 1008-1016 (2010).

44. Lu, K. T. et al. Hippocampal neurogenesis after traumatic brain injury is mediated by vascular endothelial growth factor receptor- 2 and the Raf/MEK/ ERK cascade. J. Neurotrauma 28, 441-450 (2011).

45. Li, M. et al. The role of vascular endothelial growth factor and vascular endothelial growth inhibitor in clinical outcome of traumatic brain injury. Clin. Neurol. Neurosurg. 144, 7-13 (2016).

46. Geiseler, S. J. \& Morland, C. The janus face of VEGF in stroke. Int. J. Mol. Sci. 19, 1362 (2018).

47. Miquerol, L., Gertsenstein, M., Harpal, K., Rossant, J. \& Nagy, A. Multiple developmental roles of VEGF suggested by a LacZ-tagged allele. Dev. Biol. 212, 307-322 (1999).

48. Chen, Q. et al. Endothelial cells are progenitors of cardiac pericytes and vascular smooth muscle cells. Nat. Commun. 7, 12422 (2016)

49. Seo, H., Hwang, Y., Choe, K. \& Kim, P. In vivo quantitation of injected circulating tumor cells from great saphenous vein based on video-rate confocal microscopy. Biomed. Opt. Express 6, 2158 (2015).

50. Han, S. et al. Amelioration of sepsis by TIE2 activation-induced vascular protection. Sci. Transl. Med. 8, 355ra55 (2016).

51. Kokubu, H. \& Lim, J. X-gal staining on adult mouse brain sections. Bio Protoc. 4, e1064 (2014).

52. Stuart, T. et al. Comprehensive integration of single-cell data. Cell 177, 1888-1902.e21 (2019).

53. Wolock, S. L., Lopez, R. \& Klein, A. M. Scrublet: computational identification of cell doublets in single-cell transcriptomic data. Cell Syst. 8, 281-291.e9 (2019).

54. Finak, G. et al. MAST: A flexible statistical framework for assessing transcriptional changes and characterizing heterogeneity in single-cell RNA sequencing data. Genome Biol. 16, 278 (2015).

\section{Acknowledgements}

We thank Yoshiaki Kubota (Keio University) for VE-cadherin-Cre-ER ${ }^{\mathrm{T} 2}$ mice, Hoon-Ki Sung (University of Toronto) for $V E G F-A^{+/ L a c Z}$ mice, Masanori Hirashima (Kobe University) for VEGFR2 ${ }^{\mathrm{fl} / \mathrm{fl}}$ mice, Hellmut Augustine (Heidelberg University) for Tie $2^{\mathrm{fl} / \mathrm{fl}}$, David Shima (University College London) for Dll4 $4^{\mathrm{f} / \mathrm{fl}}$ and Ralf Adams (Max-Planck Institute, Muenster) for $P D G F R \beta$-Cre-ER ${ }^{\mathrm{T} 2}$ mice. This study was supported by grants from the National Research Foundation of Korea (NRF-2018R1A2A1A05022672, I.K.), Institute for Basic Science (IBS-R025-D1-2015, G.Y.K.), and Korea Brain Research Institute (19-BR-0304, H.S.K.), all funded by the Ministry of Science and ICT, Republic of Korea.

\section{Author contributions}

B.I.K., H.J.L., G.Y.K., and I.K. conceived the study. B.I.K., H.J.L., and P.A.K. performed most of the experiments with contributions from J.H.K. and M.J.Y. M.J.Y. performed single-cell RNA sequencing. H.S.K. provided human autopsy samples. B.I.K., H.J.L., G.Y.K., and I.K. generated the figures and wrote the manuscript. G.Y.K. and I.K. supervised the study.

\section{Competing interests}

The authors have declared that no competing of interest exists.

\section{Additional information}

Supplementary information is available for this paper at https://doi.org/10.1038/s41467020-17545-2.

Correspondence and requests for materials should be addressed to G.Y.K. or I.K.

Peer review information Nature Communications thanks the anonymous reviewer(s) for their contribution to the peer review of this work.

Reprints and permission information is available at http://www.nature.com/reprints

Publisher's note Springer Nature remains neutral with regard to jurisdictional claims in published maps and institutional affiliations.

(c) (i) Open Access This article is licensed under a Creative Commons Attribution 4.0 International License, which permits use, sharing, adaptation, distribution and reproduction in any medium or format, as long as you give appropriate credit to the original author(s) and the source, provide a link to the Creative Commons license, and indicate if changes were made. The images or other third party material in this article are included in the article's Creative Commons license, unless indicated otherwise in a credit line to the material. If material is not included in the article's Creative Commons license and your intended use is not permitted by statutory regulation or exceeds the permitted use, you will need to obtain permission directly from the copyright holder. To view a copy of this license, visit http://creativecommons.org/ licenses/by/4.0/.

(C) The Author(s) 2020 